\title{
Enhanced Self-Assembly and Mechanical Properties of Cellulose-Based Triblock Copolymers: Comparisons with Amylose-Based Triblock Copolymers
}

Satoshi Katsuhara, ${ }^{a}$ Yasuko Takagi, ${ }^{a}$ Naoki Sunagawa, ${ }^{b}$ Kiyohiko Igarashi, ${ }^{b, c}$ Takuya Yamamoto, ${ }^{d}$ Kenji

Tajima, ${ }^{*} d$ Takuya Isono, ${ }^{*, d}$ Toshifumi Satoh $*, d$

${ }^{a}$ Graduate School of Chemical Sciences and Engineering, Hokkaido University, Sapporo 060-8628, Japan.

${ }^{b}$ Department of Biomaterial Sciences, Graduate School of Agriculture and Life Sciences, The University of Tokyo, Tokyo 113-8657, Japan.

${ }^{c}$ VTT Technical Research Centre of Finland Ltd., VTT FI-02044, Finland; Department of Biomaterial Sciences,

Graduate School of Agriculture and Life Sciences, The University of Tokyo, Tokyo 113-8657, Japan.

${ }^{d}$ Faculty of Engineering, Hokkaido University, Sapporo 060-8628, Japan. 


\section{*Corresponding Authors}

Kenji Tajima - Faculty of Engineering, Hokkaido University, Sapporo 060-8628, Japan; ORCID 0000-0003-37642084Email: ktajima@eng.hokudai.ac.jp

Takuya Isono - Faculty of Engineering, Hokkaido University, Sapporo 060-8628, Japan; ORCID 0000-0003-3746-2084;

Email: isono.t@eng.hokudai.ac.jp

Toshifumi Satoh - Faculty of Engineering, Hokkaido University, Sapporo 060-8628, Japan; ORCID 0000-0001-5449-9642;

Email: satoh@eng.hokudai.ac.jp

Number of pages: 43

Number of figures: 22

Number of schemes: 9 


\section{Experimental}

\section{Materials}

$\alpha$-D-Glucose 1-phosphate disodium salt hydrate $(\alpha \mathrm{G} 1 \mathrm{P}, \quad \geq 97 \%), \quad 4-(2$-hydroxyethyl)-1piperazineethanesulfonic acid (HEPES, $\geq 99.5 \%$, titration), copper(I) bromide (CuBr, 99.999\%), and 2,5dihydroxybenzonoic acid (98\%) were purchased from Sigma Aldrich and used as received. D(+)-cellobiose (CB), sodium sulfate $\left(\mathrm{Na}_{2} \mathrm{SO}_{4},>99.0 \%\right)$, anhydrous magnesium sulfate $\left(\mathrm{MgSO}_{4},>95 \%\right)$, propargylamine ( $>95 \%)$, acetic anhydride $(>97 \%)$, hydrochloric acid $(\mathrm{HCl}, 35-37 \%)$, toluene $(>99.0 \%)$, ethyl acetate (AcOEt, $>99.3 \%$ ), acetone $(>99.0 \%), N, N$-dimethylformamide (DMF, $>99.0 \%), N, N$-dimethylethanamide (DMAc, $>99.0 \%$ ), lithium chloride ( $\mathrm{LiCl},>99.0 \%)$, and dry $\mathrm{CH}_{2} \mathrm{Cl}_{2}(>99.5 \%$; water content, $<0.001 \%)$ were purchased from Kanto Chemical Co., Inc. and used as received. Dichloromethane $\left(\mathrm{CH}_{2} \mathrm{Cl}_{2},>99.0 \%\right)$ and methanol (MeOH, 99.6\%) were purchased from Junsei Chemical Co., Ltd., and used as received. $N, N, N^{\prime}, N^{\prime \prime}, N^{\prime \prime}-$ Pentamethyldiethlenetriamine (PMDETA, >98.0\%), diphenyl phosphate (DPP, >98.0\%), 1,4benzenedimethanol (BDM, >99.0\%), 1-(3-dimethylaminopropyl)-3-ethylcarbodiimide hydrochloride (EDC $\cdot \mathrm{HCl},>98.0 \%$ ), and 4-dimethylaminopyridine (DMAP, $>99.0 \%$ ) were purchased from Tokyo Chemical Industry Co., Ltd. (TCI) and used as received. FujioligoG67 $\left(\mathrm{Mal}_{n}\right)$ was supplied from Nihon Shokuhin Kako Co., Ltd., and was co-evaporated with a mixed solvent of toluene and $\mathrm{MeOH}(9 / 1(\mathrm{v} / \mathrm{v}))$ and dried under vacuum to remove water for few days before use. $\delta$-decanolactone ( $\delta$-DL) was purchased from TCI and purified by distillation over $\mathrm{CaH}_{2}$ under reduced pressure. Dowex ${ }^{\circledR} 50 \mathrm{WX} 2$ hydrogen form was purchased from Sigma Aldrich and was washed with $\mathrm{MeOH}$ before use. Cellodextrin phosphorylase (CDP) ${ }^{1}$ and 6azidohexanoic acid ${ }^{2}$ were prepared according to previous reported methods. 


\section{Instruments}

\section{${ }^{1}$ H NMR measurement}

${ }^{1} \mathrm{H}$ NMR (400 MHz) spectra were obtained using a JEOL JNM-ECS 400 instrument at $25^{\circ} \mathrm{C}$.

\section{Size exclusion chromatography (SEC)}

SEC measurements were performed at $40{ }^{\circ} \mathrm{C}$ in THF (flow rate, $1.0 \mathrm{~mL} \mathrm{~min}^{-1}$ ) using a Jasco highperformance liquid chromatography system (PU-980 Intelligent HPLC Pump, CO-2065 Plus Intelligent Column Oven, RI-2031 Plus Intelligent RI Detector, and DG-2080-53 Degasser) equipped with a Shodex KFG guard column $(4.6 \mathrm{~mm} \times 10 \mathrm{~mm}$; particle size, $8 \mu \mathrm{m})$ and two Shodex KF-804L columns (linear; particle size $7 \mu \mathrm{m} ; 8.0 \mathrm{~mm} \times 300 \mathrm{~mm}$; exclusion limit, $4 \times 10^{4}$ ) or in DMF (flow rate, $0.6 \mathrm{~mL} \mathrm{~min}{ }^{-1}$; containing 0.01 mol L ${ }^{-1}$ LiCl) using a Jasco HPLC system (PU-980 Intelligent HPLC Pump, CO-965 Column Oven, RI-930 Intelligent RI Detector, and DG-2080-53 Degasser) equipped with a Shodex KD-G guard column (4.6 mm $\times$ $10 \mathrm{~mm}$; particle size, $8 \mu \mathrm{m}$ ), a Shodex Asahipak GF-310 HQ column (linear; particle size, $5 \mu \mathrm{m}$; $7.5 \mathrm{~mm} \times$ $300 \mathrm{~mm}$; exclusion limit, $4 \times 10^{4}$ ) and a Shodex Asahipak GF-7 M HQ column (linear; particle size, $9 \mu \mathrm{m} ; 7.5$ $\mathrm{mm} \times 300 \mathrm{~mm}$; exclusion limit, $\left.1.0 \times 10^{7}\right)$. The number-average molecular weight $\left(M_{\mathrm{n}, \mathrm{SEC}}\right)$ and dispersity $(\bigoplus)$ were calculated based on polystyrene standards.

\section{Preparative SEC}

The preparative SEC purification was performed in $\mathrm{CHCl}_{3}\left(3.5 \mathrm{~mL} \mathrm{~min}^{-1}\right)$ at room temperature using LC-9201 liquid chromatography system (Japan Analytical Industry Co. Ltd. (JAI)) equipped with a JAI JAIGEL-3H column $\left(20 \mathrm{~mm} \times 600 \mathrm{~mm}\right.$; exclusion limit, $\left.7 \times 10^{4}\right)$ and a JAI RI-50s refractive index detector.

\section{Fourie transform infrared spectroscopy (FT-IR)}

The FT-IR spectra were obtained using a PerkinElmer Frontier MIR spectrometer equipped with a 
single reflection diamond universal attenuated total reflection (ATR) accessory.

\section{Differential scanning calorimetry (DSC) measurement}

The DSC experiments were performed using a Hitachi DSC 7000X under nitrogen atmosphere. All polymer samples were heated to $200{ }^{\circ} \mathrm{C}$, cooled to $-100{ }^{\circ} \mathrm{C}$, and heated again to $200{ }^{\circ} \mathrm{C}$ at the heating and cooling rate of $10^{\circ} \mathrm{C} \min ^{-1}$.

\section{Thermogravimetric analysis (TGA)}

The TGA analysis was performed using Hitachi STA200RV under nitrogen atmosphere. All polymer samples were heated up to $600{ }^{\circ} \mathrm{C}$ at the heating rate of $10^{\circ} \mathrm{C} \mathrm{min}^{-1}$.

\section{Matrix-assisted laser desorption ionization time-of-flight mass spectrometry (MALDI-TOF MS)}

The MALDI-TOF MS of the obtained products was performed using an AB Sciex TOF/TOF 5800 system equipped with a 349-nm Nd:YAG laser. Five hundred shots were accumulated for the spectra and an acceleration voltage was adjusted between 10 and $30 \mathrm{kV}$ depending on both the molecular weight and the nature of each analyzed compounds in the reflector mode. For the ethynyl-functionalized cellooligosaccharide, the sample was prepared by mixing an ultrapure water dispersion of the compound $\left(1.0 \mathrm{mg} \mathrm{mL}^{-1}, 200 \mu \mathrm{L}\right)$, acetonitrile solution of a matrix (2,5-dihydroxybenzoic acid, $\left.10 \mathrm{mg} \mathrm{mL}^{-1}, 100 \mu \mathrm{L}\right)$, and trifluoroacetic acid $(0.2 \%(\mathrm{v} / \mathrm{v}), 300 \mu \mathrm{L})$. A $1 \mu \mathrm{L}$ aliquot of this mixture was loaded on a sample plate. For ethynyl-functionalized cellooligosaccharide acetate, the ethynyl-functionalized maltooligosaccharide, and ethynyl-functionalized maltooligosaccharide acetate, the samples were prepared by mixing a THF solution of the compound (5 mg $\left.\mathrm{mL}^{-1}, 100 \mu \mathrm{L}\right)$ and a matrix $\left(2,5\right.$-dihydroxybenzoic acid, $\left.10 \mathrm{mg} \mathrm{mL}^{-1}, 500 \mu \mathrm{L}\right)$. A $1 \mu \mathrm{L}$ aliquot of this mixture was loaded on a sample plate, which was coated by an acetone solution of $\mathrm{NaI}\left(1 \mu \mathrm{L}, 1 \mathrm{mmol} \mathrm{L}^{-1}\right)$ as the cationic agent. 


\section{Atomic force microscopy (AFM) observation}

The AFM phase images were obtained using a Molecular Imaging PicoPlus atomic force microscope operating in the tapping mode with a silicon cantilever (Nanoworld AG, NANOSENSORS ${ }^{\mathrm{TM}}$ PPP-NCH) having resonant frequency and spring constant of $190 \mathrm{kHz}$ and $48 \mathrm{~N} \mathrm{~m}^{-1}$, respectively. The thin films for the AFM observation were prepared by spin-coating (2000 rpm for $60 \mathrm{~s})$ the polymer solution in toluene $(1 \%$ $(w / w))$ onto a Si substrate with a native oxide layer.

\section{Small-angle X-ray scattering (SAXS) and wide-angle X-ray diffraction (WAXD)}

The SAXS and WAXD measurements of the obtained polymers were performed at the BL-6A beamline of the Photon Factory in the High Energy Accelerator Research Organization (KEK, Tsukuba, Japan) using X-ray beams with $\lambda=0.15 \mathrm{~nm}$ at room temperature. The scattering data were collected by a 2D detector (PILATUS3 1M (SAXS) (Dectris Ltd.)), where the samples-to-detector distance was set to be $1.5 \mathrm{~m}$ for SAXS measurement. The scattering angle $(\theta)$ was calibrated using silver behenate (Nagara Science Co., Ltd) as the standard and derived the scattering vector $(q)$ from Bragg's equation $(|q|=(4 \pi / \lambda) \sin (\theta / 2))$. The domain-spacing (d) value was calculated by $d=2 \pi / q^{*}$ ( $q^{*}$ is principal scattering peak position). The polymer films or powders were sandwiched by two pieces of Kapton tapes with a spacer of a stainless washer, which were applied for the measurement.

\section{Tensile testing}

The tensile tests were performed with an EZ-SX (SHIMADZU CO.) tensile tester at the temperature of $23{ }^{\circ} \mathrm{C}$ and the humidity of $55 \%$. The film samples for the tensile tests were prepared by casting the polymer solutions from the toluene solution $\left(20.0 \%(\mathrm{w} / \mathrm{w})\right.$ for $\mathrm{AcCel}_{n}-b-\mathrm{PDL}-b-\mathrm{AcCel}_{n}$ and $30.0 \%(\mathrm{w} / \mathrm{w})$ for $\mathrm{AcMal}_{n^{-}}$ $b$-PDL- $b-\mathrm{AcMal}_{n}$ ) on a Teflon dish and drying at r.t. for $15 \mathrm{~h}$ followed by vacuum drying at room temperature 
for 1 day. After drying, the film samples were cut into a dog bone shape of $12 \times 3 \times 0.05-0.15 \mathrm{~mm}$. For each polymer, five samples were tested and the average values of the elastic modulus, strain at break, stress at break, and toughness were calculated. The crosshead speed applied during the measurements was $10 \mathrm{~mm} \mathrm{~min}^{-1}$. The strain at break was taken as the engineering strain where the stress drops suddenly. 


\section{Synthesis of ethynyl-functionalized cellobiose $(\mathrm{CB}-\mathrm{C} \equiv \mathrm{CH})^{3}$}

Scheme S1. Synthesis of CB-C $\equiv \mathrm{CH}$

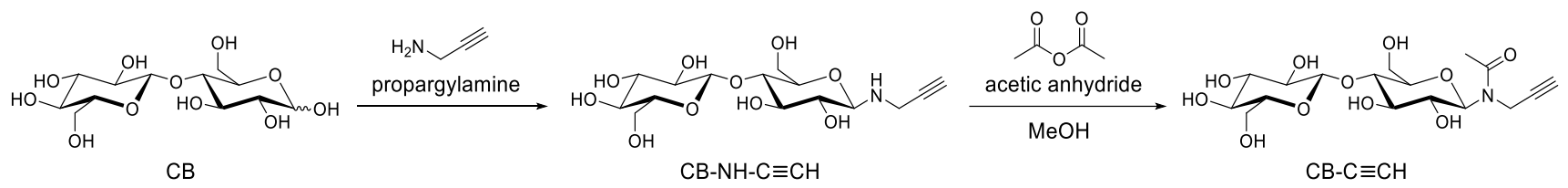

CB (10.00 g, $14.6 \mathrm{mmol})$ and propargylamine $(20.7 \mathrm{~mL}, 323 \mathrm{mmol})$ were added to a round-bottom

flask, and the mixture was stirred for 5 days under a nitrogen atmosphere. The mixture was co-evaporated with a mixed solvent of toluene and $\mathrm{MeOH}(9 / 1(\mathrm{v} / \mathrm{v}))$ to remove the excessive propargylamine. The obtained product was dissolved in $\mathrm{MeOH}$ and then precipitated in $\mathrm{CH}_{2} \mathrm{Cl}_{2}$. The precipitate was filtrated and washed with a mixture of $\mathrm{MeOH}$ and $\mathrm{CH}_{2} \mathrm{Cl}_{2}(1 / 4(\mathrm{v} / \mathrm{v}), 100 \mathrm{~mL})$. A solution of acetic anhydride in $\mathrm{MeOH}(1 / 20(\mathrm{v} / \mathrm{v})$, $480 \mathrm{~mL}$ ) was added to the obtained precipitate and the whole mixture was stirred for $20 \mathrm{~h}$ at room temperature. After the reaction, the solvent was subjected to rotary evaporation, followed by co-evaporation of acetic anhydride with a mixed solvent of toluene and $\mathrm{MeOH}(1 / 1(\mathrm{v} / \mathrm{v}))$ to give $\mathrm{CB}-\mathrm{C} \equiv \mathrm{CH}$ as a white solid $(11.1 \mathrm{~g})$. Yield: $89.3 \%$.

${ }^{1} \mathrm{H}$ NMR (400 MHz, $\left.\mathrm{D}_{2} \mathrm{O}\right): \delta(\mathrm{ppm}) 5.52,5.07(2 \mathrm{x} \mathrm{d}$, rotamers, $-\mathrm{C} H \mathrm{~N}(\mathrm{Ac})-), 4.50\left(\mathrm{~d}, J=7.6 \mathrm{~Hz}, H-1^{\mathrm{CB}}\right)$, 4.31-3.25 (m, H-2, -3, -4, -5, -6 $\left.6^{\mathrm{CB}}, \mathrm{O} H^{\mathrm{CB}},-\mathrm{N}(\mathrm{Ac}) \mathrm{CH}_{2}-\right), 2.71,2.55$ (2x s, rotamers, $\left.-\mathrm{CC} H\right), 2.29,2.21(2 \mathrm{x} \mathrm{s}$, rotamers, $\left.-\mathrm{N}(\mathrm{C}=\mathrm{O}) \mathrm{CH}_{3}\right)$. 
(a)

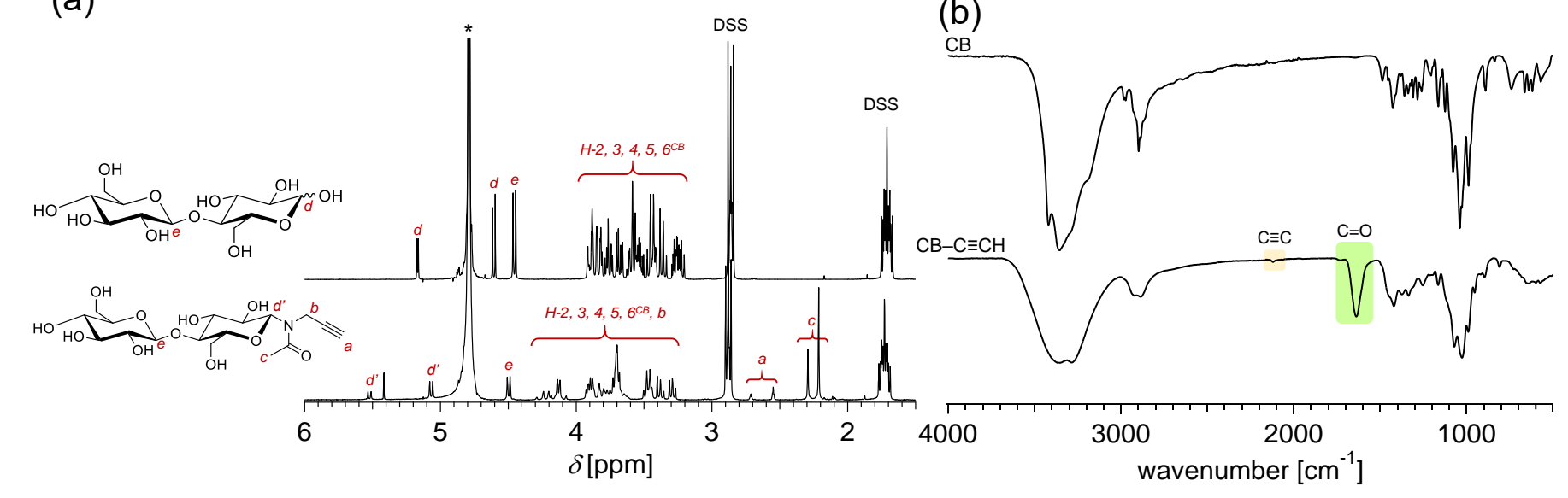

Figure S1. (a) ${ }^{1} \mathrm{H}$ NMR spectra $\left(400 \mathrm{MHz}, \mathrm{D}_{2} \mathrm{O}\right)$ and (b) FT-IR spectra of $\mathrm{CB}$ (upper) and $\mathrm{CB}-\mathrm{C} \equiv \mathrm{CH}$ (lower). 
Scheme S2. Synthesis of $\mathrm{Cel}_{n}-\mathrm{C} \equiv \mathrm{CH}$

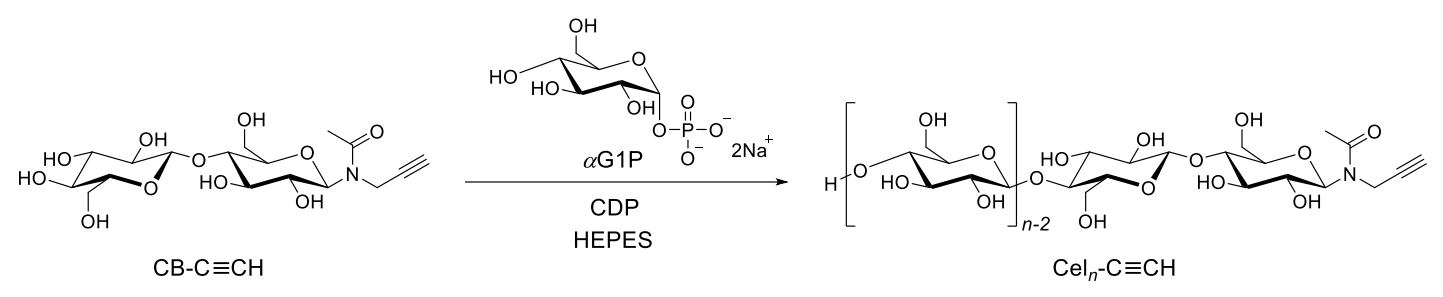

$200 \mathrm{mM}$ D-glucose 1-phosphate $(\alpha \mathrm{G} 1 \mathrm{P})$ and $50 \mathrm{mM} \mathrm{CB}-\mathrm{C} \equiv \mathrm{CH}$ was incubated with $10 \mathrm{mg} \mathrm{L}^{-1} \mathrm{CDP}$ in $500 \mathrm{mM}$ 4-(2-hydroxyethyl)-1-piperazineethanesulfonic acid (HEPES) buffer solutions (pH 7.5, $300 \mathrm{~mL}$, divided into 30 centrifuge tubes) at $60{ }^{\circ} \mathrm{C}$. After incubating for 7 days, the reaction mixtures were centrifuged to isolate the insoluble product and the resulting pellet was washed with water purified by reverse osmosis filters (RO water) followed by centrifugation at $13,000 \mathrm{rpm}$ for $10 \mathrm{~min}$ at $4{ }^{\circ} \mathrm{C}$ several times to give swollen $\mathrm{Cel}_{n}-\mathrm{C} \equiv \mathrm{CH}$ with water. The aliquot of the obtained product was dried by vacuum to calculate the yield (3.19 g). Yield: $23.0 \%$.

$M_{\mathrm{n}, \mathrm{NMR}}=1,260 \mathrm{~g} \mathrm{~mol}^{-1}, M_{\mathrm{n}, \mathrm{MALDI}}=1,190 \mathrm{~g} \mathrm{~mol}^{-1}$.

${ }^{1} \mathrm{H}$ NMR $\left(400 \mathrm{MHz}, 10 \%(\mathrm{w} / \mathrm{w}) \mathrm{NaOD}-\mathrm{D}_{2} \mathrm{O}\right): \delta(\mathrm{ppm}) 5.39,4.88(2 \mathrm{x}$ d, rotamers, $-\mathrm{C} H \mathrm{~N}(\mathrm{Ac})-), 4.48(\mathrm{~m}, H-$ $\left.1^{\mathrm{Cel} n}\right), 4.31-3.21\left(\mathrm{~m}, H-2,-3,-4,-5,-6^{\mathrm{Cel} n}, \mathrm{O} H^{\mathrm{Cel} n},-\mathrm{N}(\mathrm{Ac}) \mathrm{CH}_{2}-\right), 2.54(\mathrm{~m},-\mathrm{CCH}), 2.30,2.23(2 \mathrm{x} \mathrm{m}$, $\left.-\mathrm{N}(\mathrm{C}=\mathrm{O}) \mathrm{CH}_{3}\right)$. 


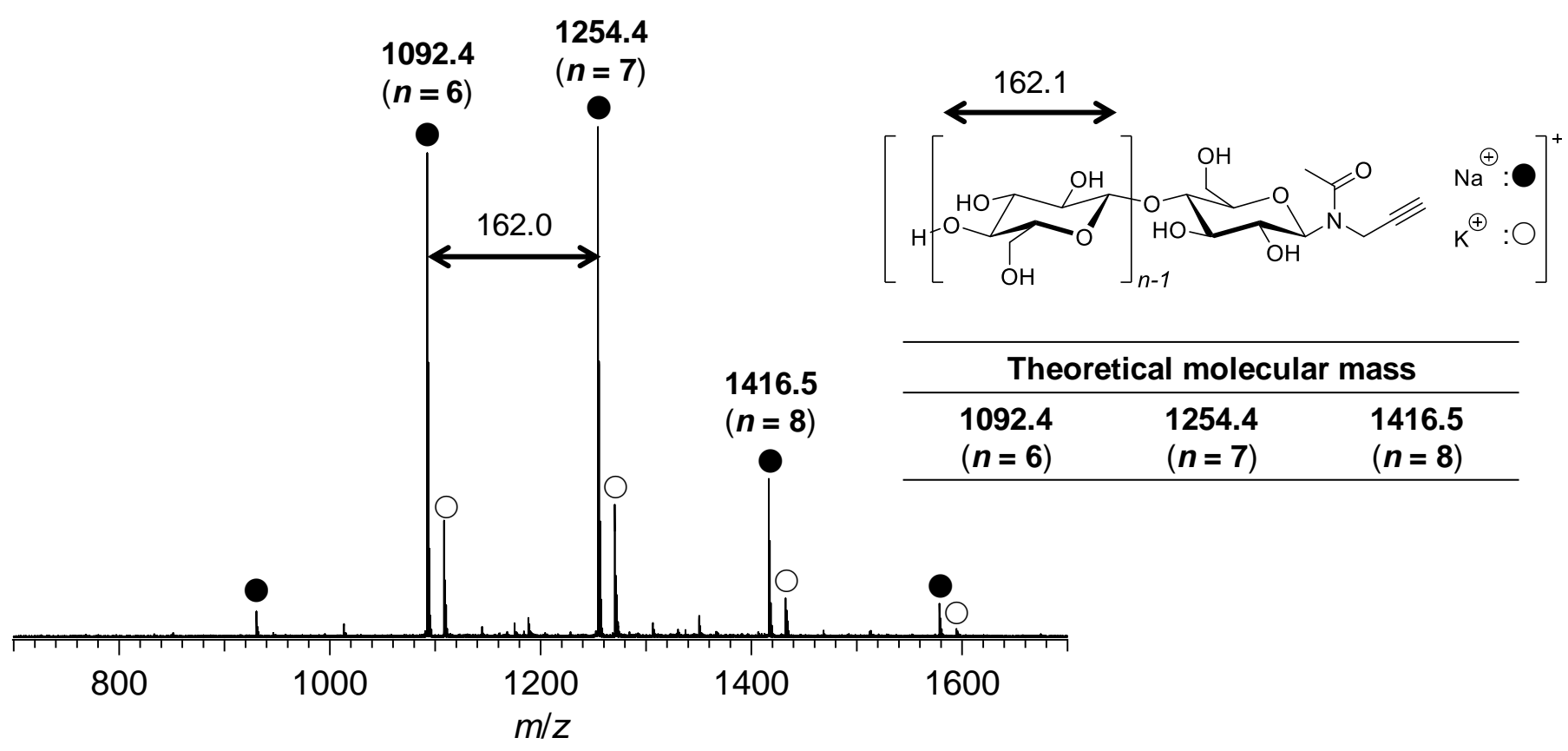

Figure S2. MALDI-TOF mass spectrum and theoretical molecular mass of $\mathrm{Cel}_{n}-\mathrm{C} \equiv \mathrm{CH}$ 


\section{Synthesis of ethynyl-functionalized cellooligosaccharide acetate $\left(\mathrm{AcCel}_{n}-\mathrm{C} \equiv \mathrm{CH}\right)$}

Scheme S3. Synthesis of $\mathrm{AcCel}_{n}-\mathrm{C} \equiv \mathrm{CH}$
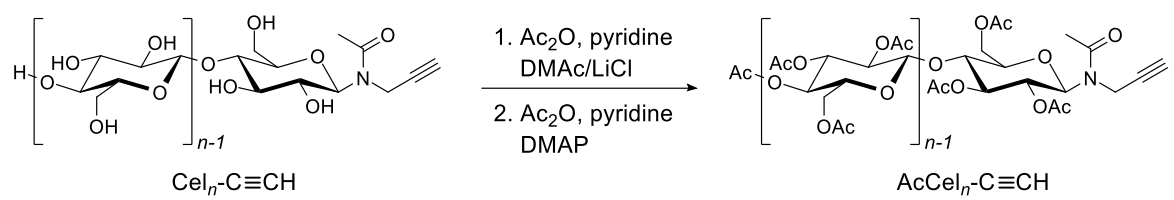

Wet $\mathrm{Cel}_{n}-\mathrm{C} \equiv \mathrm{CH}(3.19 \mathrm{~g}$, by dry weight) was dispersed in acetone $(40 \mathrm{~mL})$ and centrifuged twice $\left(7,000 \mathrm{rpm} / 30 \mathrm{~min} / 0{ }^{\circ} \mathrm{C}, 7,000 \mathrm{rpm} / 15 \mathrm{~min} / 0{ }^{\circ} \mathrm{C}\right)$. The pellet was dispersed in acetone $(100 \mathrm{~mL})$ and stirred over night at room temperature. After the centrifugation $\left(7,000 \mathrm{rpm} / 15 \mathrm{~min} / 0{ }^{\circ} \mathrm{C}\right)$, the pellet was washed with DMAc $(40 \mathrm{~mL})$ followed by centrifugation $\left(7,000 \mathrm{rpm} / 15 \mathrm{~min} / 0{ }^{\circ} \mathrm{C}\right)$. The pellet was dispersed in DMAc $(40$ $\mathrm{mL})$ and stirred at room temperature. After $3 \mathrm{~h}, \mathrm{LiCl}(2.14 \mathrm{~g}, 50.5 \mathrm{mmol})$ was added to the mixture and kept stirring over-night. After adding a solution of acetic anhydride in pyridine (1/1 (v/v), $20 \mathrm{~mL})$, the mixture was stirred 5 more days. The reaction mixture was diluted with $\mathrm{CH}_{2} \mathrm{Cl}_{2}$ and washed with dilute $\mathrm{HCl}(100 \mathrm{~mL})$ three times. The organic layer was dried over $\mathrm{MgSO}_{4}$ and concentrated to give a white solid. The obtained solid (3.02 g) and DMAP (76 mg, $0.62 \mathrm{mmol})$ was added to a solution of acetic anhydride in pyridine $(1 / 2$ $(\mathrm{v} / \mathrm{v}), 60 \mathrm{~mL})$ and stirred at room temperature for $70 \mathrm{~h}$. After removing the solvent by evaporation, the residue was dissolved in ethyl acetate and washed with $1 \mathrm{M} \mathrm{HCl}$ and saturated $\mathrm{NaHCO}_{3}$ solutions. The organic layer was dried over $\mathrm{Na}_{2} \mathrm{SO}_{4}$ and concentrated to give $\mathrm{AcCel}_{n}-\mathrm{C} \equiv \mathrm{CH}$ as a pale yellow solid (3.03 g). Yield: 55.0\%. $M_{\mathrm{n}, \mathrm{NMR}}=2,090 \mathrm{~g} \mathrm{~mol}^{-1}, M_{\mathrm{n}, \mathrm{MALDI}}=2,100 \mathrm{~g} \mathrm{~mol}^{-1}, M_{\mathrm{n}, \mathrm{SEC}}=2,080 \mathrm{~g} \mathrm{~mol}^{-1}(\mathrm{THF}), Ð=1.06(\mathrm{THF})$. ${ }^{1} \mathrm{H}$ NMR (400 MHz, $\mathrm{CDCl}_{3}$ ): $\delta$ (ppm) 5.84, 5.65 (2x d, rotamers, $-\mathrm{CHN}(\mathrm{Ac})-$ ), 5.30-4.76, 4.60-4.31, 4.25$3.47\left(\mathrm{~m}, \mathrm{H}-1,-2,-3,-4,-5,-6^{\mathrm{AcCel} n},-\mathrm{N}(\mathrm{Ac}) \mathrm{CH}_{2}-\right), 2.58,2.56(2 \mathrm{x}$ s, rotamers, $-\mathrm{CCH}), 2.40-1.76\left(\mathrm{~m}, \mathrm{CH}_{3^{-}}\right.$ $\left.\mathrm{Ac}^{\mathrm{AcCel} n},-\mathrm{N}(\mathrm{C}=\mathrm{O}) \mathrm{CH}_{3}\right)$. 
(a)
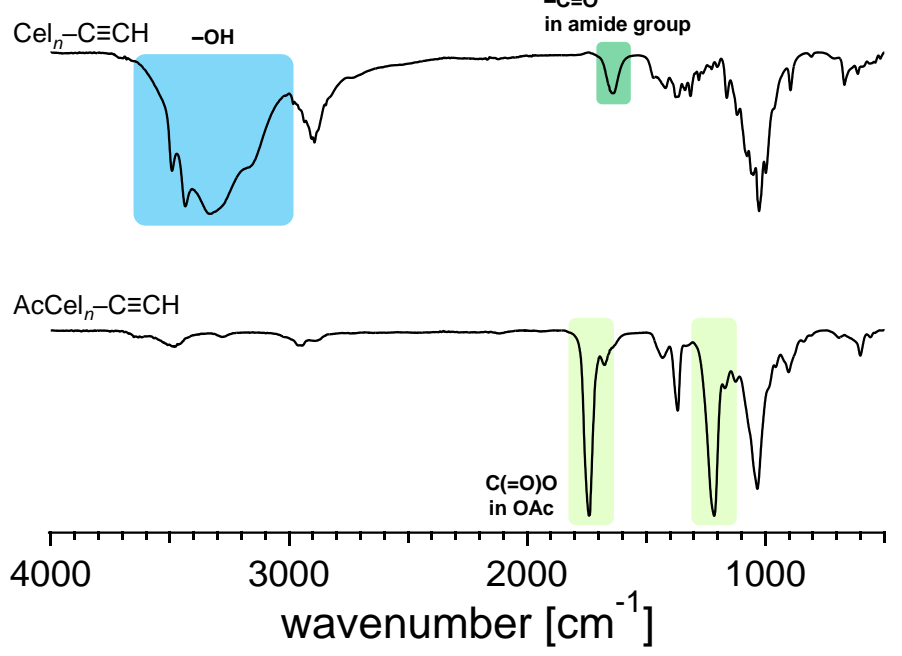

(b)

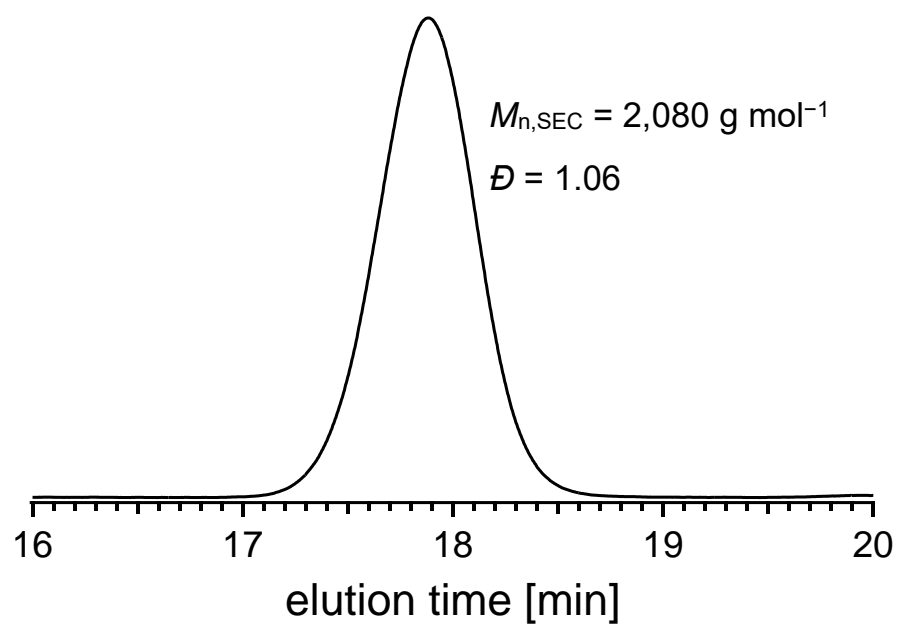

Figure S3. (a) FT-IR spectra of $\mathrm{Cel}_{n}-\mathrm{C} \equiv \mathrm{CH}$ (upper) and $\mathrm{AcCel}_{n}-\mathrm{C} \equiv \mathrm{CH}$ (lower) and (b) SEC trace of

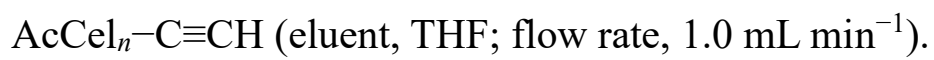


Scheme S4. Synthesis of $\mathrm{Mal}_{n}-\mathrm{C} \equiv \mathrm{CH}$

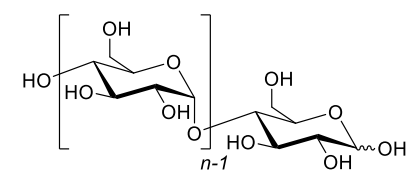

$\operatorname{Mal}_{n}(n=6 \sim 7)$

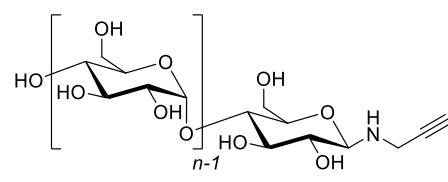

$\mathrm{Mal}_{n}-\mathrm{NH}-\mathrm{C} \equiv \mathrm{CH}$

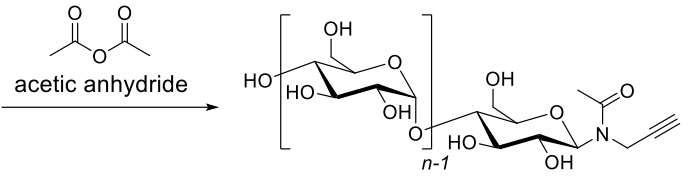

$\mathrm{Mal}_{n}-\mathrm{C} \equiv \mathrm{CH}$

$\operatorname{Mal}_{n}(14.0 \mathrm{~g})$ and propargylamine $(15.6 \mathrm{~mL}, 243 \mathrm{mmol})$ were added to a round-bottom flask and the mixture was stirred for 5 days under the nitrogen atmosphere. The mixture was diluted with $\mathrm{MeOH}$ to remove the insoluble part with the high molecular weight, and the soluble part was then poured into $\mathrm{CH}_{2} \mathrm{Cl}_{2}$. The resulting precipitate was added to a solution of acetic anhydride in $\mathrm{MeOH}(1 / 20(\mathrm{v} / \mathrm{v}), 1050 \mathrm{~mL})$ and stirred for $45 \mathrm{~h}$ at room temperature. After the solvent removal, acetic anhydride was removed by co-evaporation with a mixed solvent of toluene and $\mathrm{MeOH}(1 / 1(\mathrm{v} / \mathrm{v}))$ followed by precipitation into $\mathrm{CH}_{2} \mathrm{Cl}_{2}$. The precipitate was then dissolved in $\mathrm{MeOH}$ and poured into $\mathrm{EtOH}$ to give $\mathrm{Mal}_{n}-\mathrm{C} \equiv \mathrm{CH}$ as a white solid (3.21 g). Yield:

$21.2 \%$.

$M_{\mathrm{n}, \mathrm{NMR}}=1,070 \mathrm{~g} \mathrm{~mol}^{-1}, M_{\mathrm{n}, \mathrm{MALDI}}=1,080 \mathrm{~g} \mathrm{~mol}^{-1}, M_{\mathrm{n}, \mathrm{SEC}}=2,650 \mathrm{~g} \mathrm{~mol}^{-1}(\mathrm{DMF}), Ð=1.11(\mathrm{DMF})$.

${ }^{1} \mathrm{H}$ NMR (400 MHz, $\left.\mathrm{D}_{2} \mathrm{O}\right): \delta(\mathrm{ppm}) 5.51,5.06$ (2x d, rotamers, -CHN(Ac)-), 5.43-5.30 (br, H-1 $\left.{ }^{\mathrm{Maln}}\right), 4.31-$ $3.30\left(\mathrm{~m}, H-2,-3,-4,-5,-6^{\mathrm{Mal} n}, \mathrm{O} H^{\mathrm{Mal} n},-\mathrm{N}(\mathrm{Ac}) \mathrm{CH}_{2}-\right), 2.39,2.21\left(2 \mathrm{x}\right.$ s, rotamers, $\left.-\mathrm{N}(\mathrm{C}=\mathrm{O}) \mathrm{CH}_{3}\right)$. 

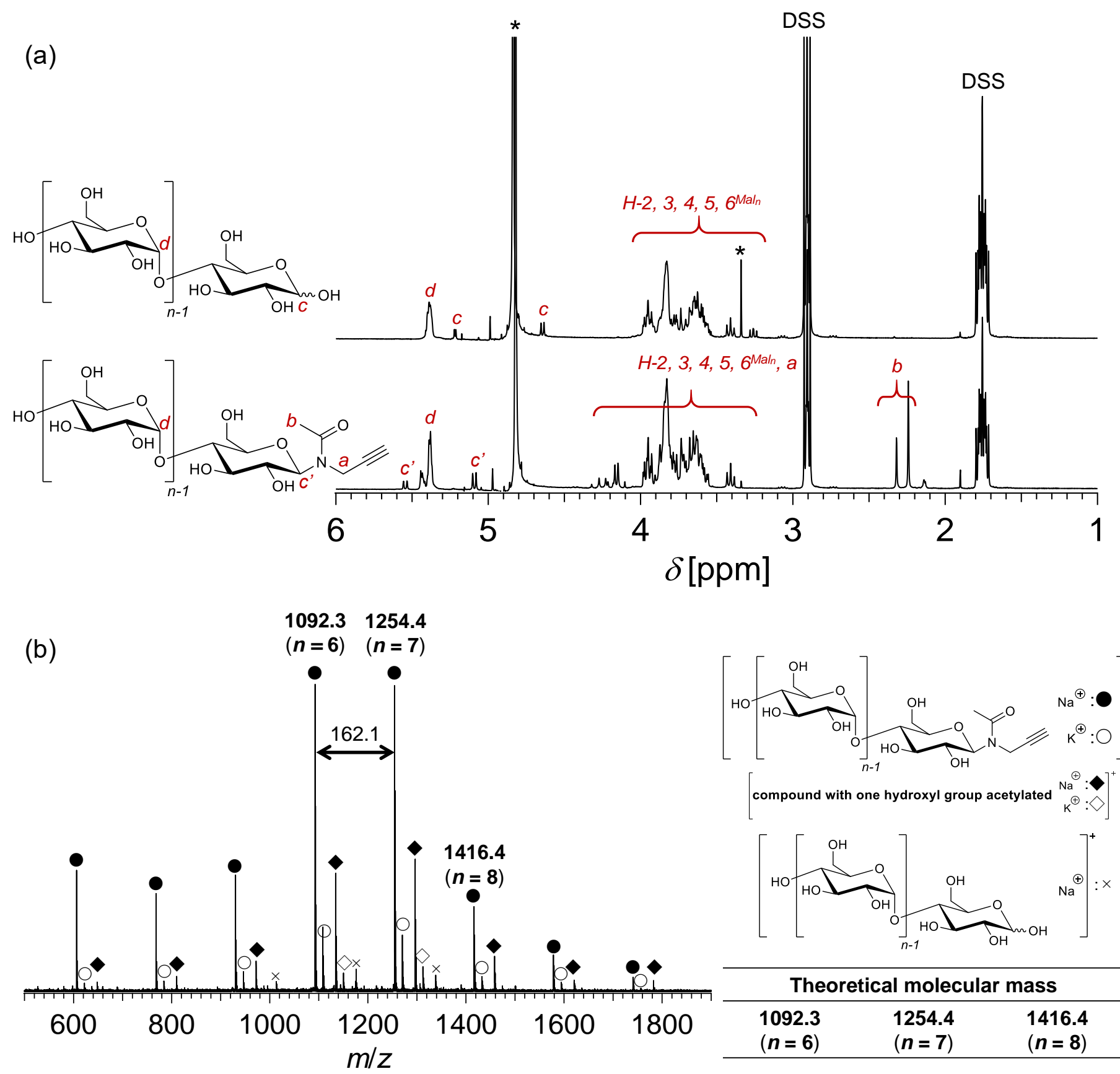

Figure S4. Characterization of $\mathrm{Mal}_{n}-\mathrm{C} \equiv \mathrm{CH}$. (a) ${ }^{1} \mathrm{H}$ NMR spectra of $\mathrm{Mal}_{n}$ (upper) and $\mathrm{Mal}_{n}-\mathrm{C} \equiv \mathrm{CH}\left(\mathrm{D}_{2} \mathrm{O}, 400\right.$ $\mathrm{MHz}$ ). (b) MALDI-TOF mass spectrum and theoretical molecular mass. 
Scheme S5. Synthesis of $\mathrm{AcMal}_{n}-\mathrm{C} \equiv \mathrm{CH}$

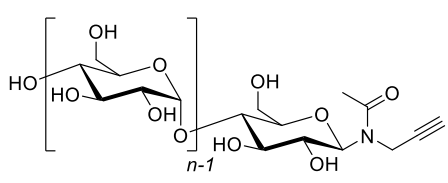

$\mathrm{Mal}_{n}-\mathrm{C} \equiv \mathrm{CH}$

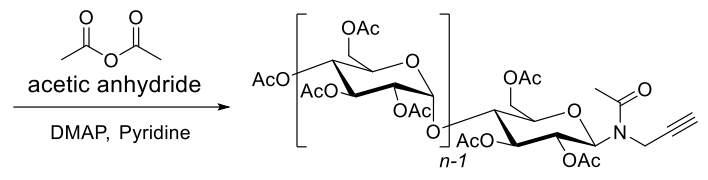

$\mathrm{AcMal}_{n}-\mathrm{C} \equiv \mathrm{CH}$

$\mathrm{Mal}_{n}-\mathrm{C} \equiv \mathrm{CH}(3.50 \mathrm{~g}, 3.27 \mathrm{mmol})$ and DMAP $(76 \mathrm{mg}, 0.62 \mathrm{mmol})$ were added to a solution of acetic anhydride in pyridine $(1 / 2(\mathrm{v} / \mathrm{v}), 105 \mathrm{~mL})$ and stirred for $70 \mathrm{~h}$ at room temperature. After removing the solvent by evaporation, the residue was dissolved in ethyl acetate and washed with $1 \mathrm{M} \mathrm{HCl}$, saturated $\mathrm{NaHCO}_{3}$, and water. The organic layer was dried over $\mathrm{Na}_{2} \mathrm{SO}_{4}$ and concentrated by evaporation. The product was purified by preparative $\mathrm{SEC}$ in $\mathrm{CHCl}_{3}$ to remove low molecular weight fraction, to afford $\mathrm{AcMal}_{n}-\mathrm{C} \equiv \mathrm{CH}$ as a pale yellow solid (2.22 g). Yield: 38.8\%.

$M_{\mathrm{n}, \mathrm{NMR}}=2,020 \mathrm{~g} \mathrm{~mol}^{-1}, M_{\mathrm{n}, \mathrm{MALDI}}=1,950 \mathrm{~g} \mathrm{~mol}^{-1}, M_{\mathrm{n}, \mathrm{SEC}}=1,490 \mathrm{~g} \mathrm{~mol}^{-1}(\mathrm{THF}), Ð=1.09(\mathrm{THF})$.

${ }^{1} \mathrm{H}$ NMR (400 MHz, $\left.\mathrm{CDCl}_{3}\right): \delta(\mathrm{ppm})$ 6.22, 5.91 (2x d, rotamers, $-\mathrm{C} H \mathrm{~N}(\mathrm{Ac})-$ ), 5.65-3.64 (m, $H-2,-3,-4,-5$, $\left.-6^{\mathrm{AcMal} n},-\mathrm{N}(\mathrm{Ac}) \mathrm{CH}_{2}-\right), 2.37-1.80\left(\mathrm{~m}, \mathrm{CH}_{3}-\mathrm{Ac}^{\mathrm{AcMal} n},-\mathrm{N}(\mathrm{C}=\mathrm{O}) \mathrm{CH}_{3}\right)$. 
(a)
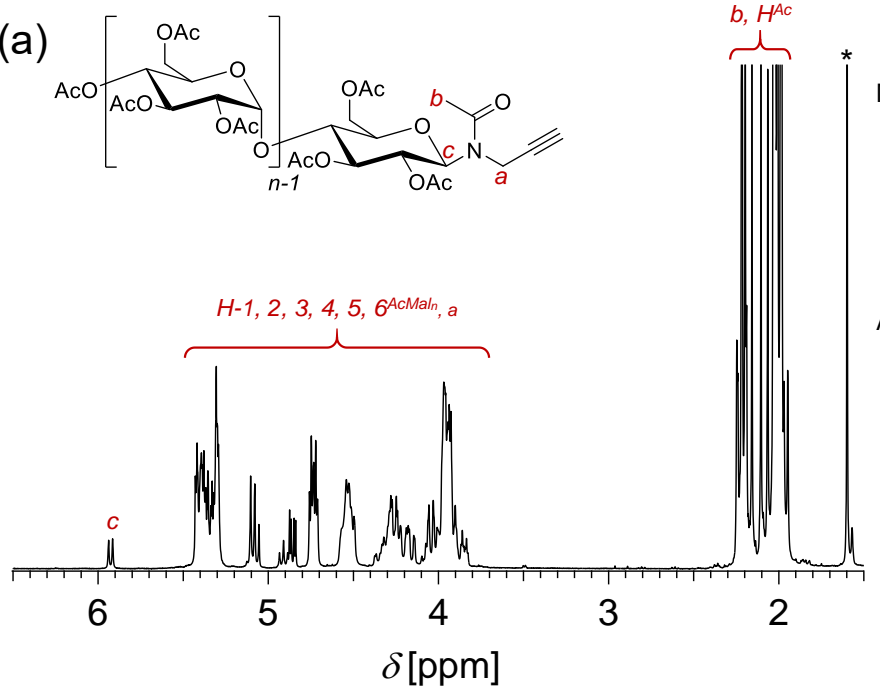

$\delta$ [ppm]

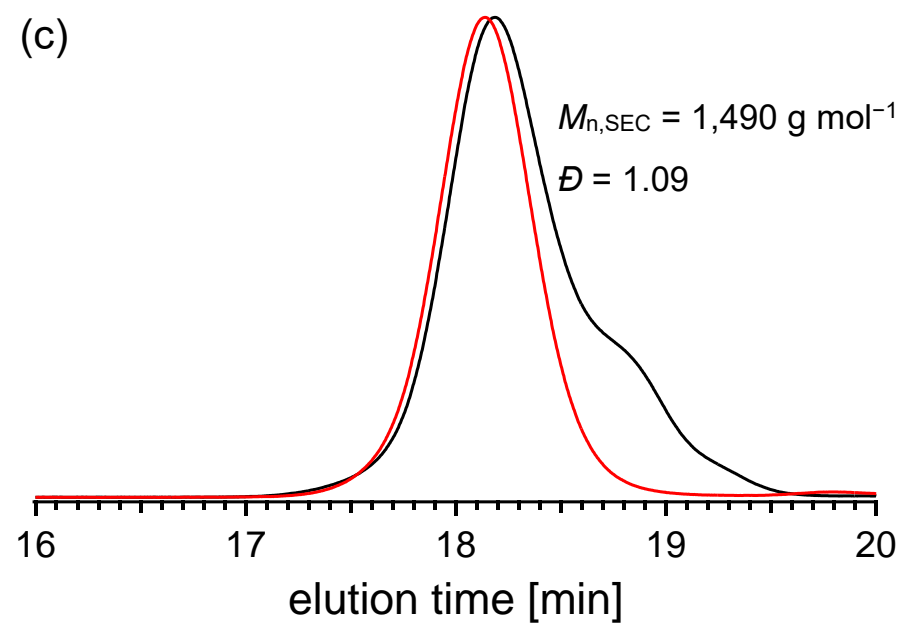

(d)

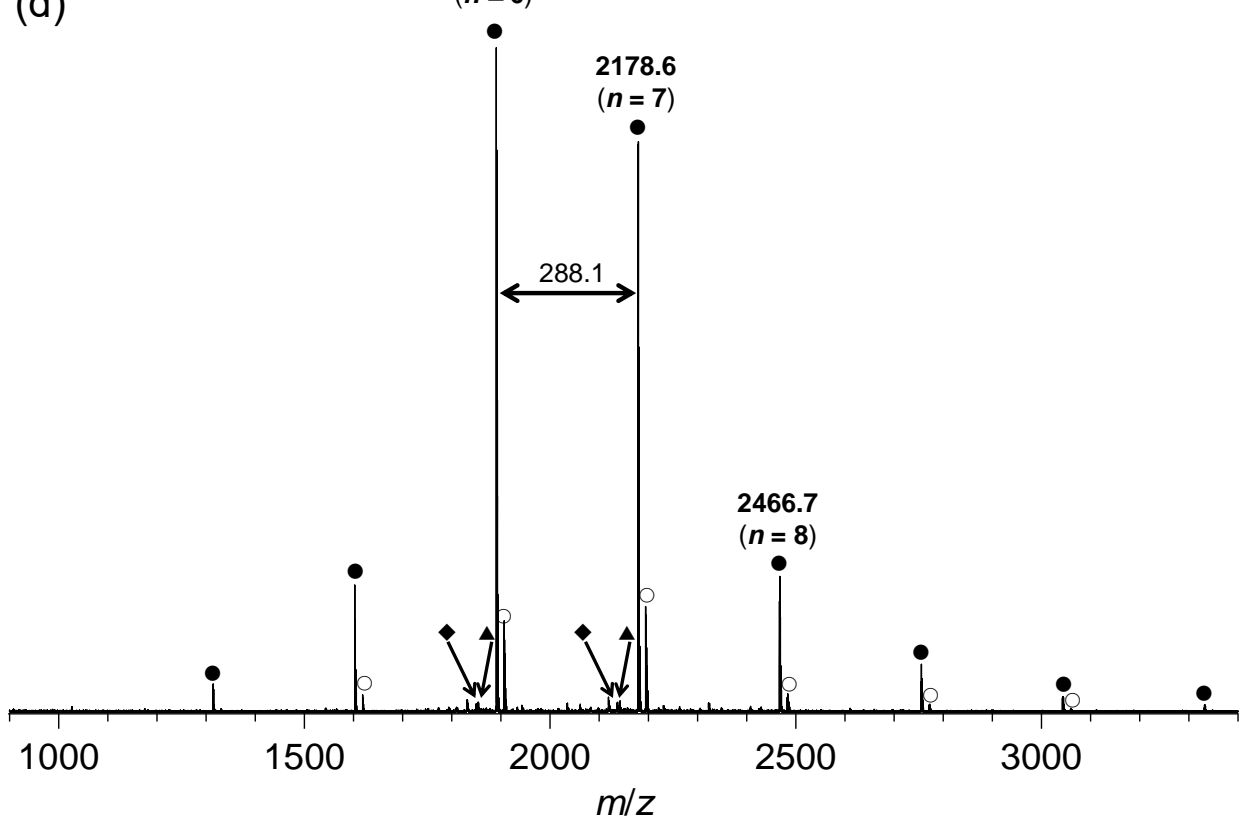

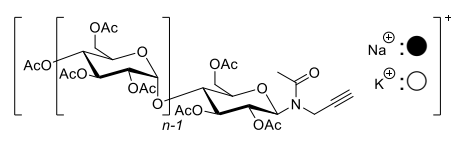

$\left[\text { AcMal- } \mathrm{C} \equiv \mathrm{CH}-\mathrm{Ac}+\mathrm{H}+\mathrm{Na}^{+}\right]^{+}$

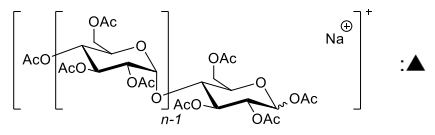

Figure S5. Characterization of $\mathrm{AcMal}_{n}-\mathrm{C} \equiv \mathrm{CH}$. (a) ${ }^{1} \mathrm{H}$ NMR spectrum $\left(\mathrm{CDCl}_{3}, 400 \mathrm{MHz}\right)$. (b) FT-IR spectra of $\mathrm{Mal}_{n}-\mathrm{C} \equiv \mathrm{CH}$ (upper) and $\mathrm{AcMal}_{n}-\mathrm{C} \equiv \mathrm{CH}$ (lower). (c) $\mathrm{SEC}$ trace of $\mathrm{AcMal}_{n}-\mathrm{C} \equiv \mathrm{CH}$ before (black) and after (red) preparative SEC purification (eluent, THF; flow rate, $1.0 \mathrm{~mL} \mathrm{~min}^{-1}$. (d) MALDI-TOF mass spectrum and theoretical molecular mass. 
Scheme S6. Synthesis of HO-PDL-OH

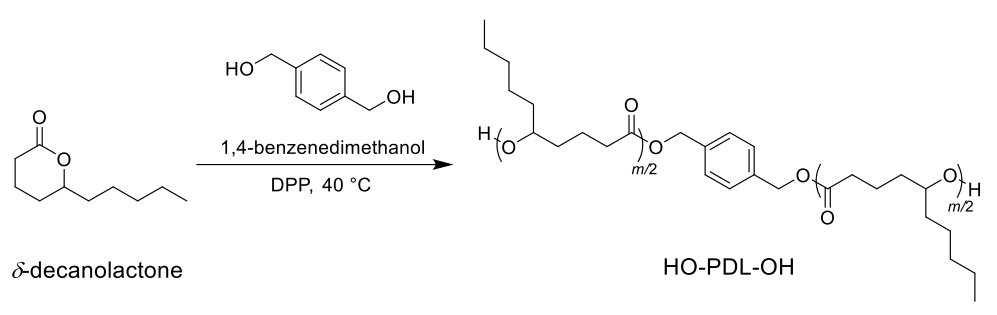

\section{Synthesis of HO-PDL6k-OH}

Typical polymerization procedure is as follows (Method A). In an argon-filled glovebox, $\delta$-DL (10.0 g, $58.7 \mathrm{mmol})$, 1,4-benzenedimethanol (199 mg, $1.44 \mathrm{mmol})$, and DPP (360 mg, $1.44 \mathrm{mmol})$ were placed in a Schlenk flask. The Schlenk flask was sealed with a septum and taken out from the glovebox. The whole mixture was stirred at $40{ }^{\circ} \mathrm{C}$ under an argon atmosphere in an oil bath. After stirring for $9 \mathrm{~h}$, the monomer conversion was reached $73 \%$ as revealed by ${ }^{1} \mathrm{H}$ NMR analysis of the aliquot of the mixture. The polymerization mixture was poured into $\mathrm{MeOH}$, and the precipitated product was washed with $\mathrm{MeOH}$ several times to remove the unreacted monomer and catalyst. Finally, $\mathrm{HO}-\mathrm{PDL}_{6 \mathrm{k}}-\mathrm{OH}$ was obtained as a colorless viscous liquid (7.80 g). Yield: $76.5 \%$.

$M_{\mathrm{n}, \mathrm{NMR}}=5,590 \mathrm{~g} \mathrm{~mol}^{-1}, M_{\mathrm{n}, \mathrm{SEC}}=8,770 \mathrm{~g} \mathrm{~mol}^{-1}(\mathrm{THF}), Ð=1.07(\mathrm{THF})$.

${ }^{1} \mathrm{H}-\mathrm{NMR}\left(400 \mathrm{MHz}, \mathrm{CDCl}_{3}\right): \delta$ (ppm) 7.28 (s, aromatic), 5.03 (s, - $\mathrm{Ph}-\mathrm{CH}_{2} \mathrm{O}-$ ), 4.80 (quin, main chain $\left.{ }^{-} \mathrm{CH}_{2} \mathrm{CH}\left(\mathrm{C}_{5} \mathrm{H}_{11}\right) \mathrm{O}^{-}\right)$, 3.57-3.47 (br, $\omega$-chain end $\left.-\mathrm{CH}_{2} \mathrm{CH}\left(\mathrm{C}_{5} \mathrm{H}_{11}\right) \mathrm{OH}\right), 2.34-2.14\left(\mathrm{~m},-(\mathrm{C}=\mathrm{O}) \mathrm{CH}_{2} \mathrm{CH}_{2}^{-}\right)$, 1.76-1.32 (m, $\left.-(\mathrm{C}=\mathrm{O}) \mathrm{CH}_{2} \mathrm{CH}_{2} \mathrm{CH}_{2} \mathrm{CH}\left(\mathrm{CH}_{2}\left(\mathrm{CH}_{2}\right)_{3} \mathrm{CH}_{3}\right) \mathrm{O}^{-}\right)$, $1.31-1.10$ (br, $\left.-\mathrm{CH}\left(\mathrm{CH}_{2}\left(\mathrm{CH}_{2}\right)_{3} \mathrm{CH}_{3}\right) \mathrm{O}^{-}\right), 0.87$ $\left(\mathrm{t}, J=6.6 \mathrm{~Hz},-\mathrm{CH}_{3}\right)$. 


\section{Synthesis of HO-PDL $10 \mathrm{k}-\mathrm{OH}$}

The Method A was used for the polymerization of $\delta$-DL $(10.0 \mathrm{~g}, 58.7 \mathrm{mmol})$ with 1,4benzenedimethanol $(98 \mathrm{mg}, 0.71 \mathrm{mmol})$ and $\mathrm{DPP}(178 \mathrm{mg}, 0.710 \mathrm{mmol})$ to give $\mathrm{HO}-\mathrm{PDL}_{10 \mathrm{k}}-\mathrm{OH}$ as a colorless viscous liquid (6.29 g). Yield: $62.3 \%$.

$M_{\mathrm{n}, \mathrm{NMR}}=10,000 \mathrm{~g} \mathrm{~mol}^{-1}, M_{\mathrm{n}, \mathrm{SEC}}=12,800 \mathrm{~g} \mathrm{~mol}^{-1}(\mathrm{THF}), Ð=1.06(\mathrm{THF})$.

${ }^{1} \mathrm{H}-\mathrm{NMR}\left(400 \mathrm{MHz}, \mathrm{CDCl}_{3}\right): \delta$ (ppm) 7.34 (s, aromatic), $5.10\left(\mathrm{~s},-\mathrm{Ph}-\mathrm{CH}_{2} \mathrm{O}-\right.$ ), 4.87 (quin, main chain $\left.-\mathrm{CH}_{2} \mathrm{CH}\left(\mathrm{C}_{5} \mathrm{H}_{11}\right) \mathrm{O}^{-}\right)$, 3.64-3.54 (m, $\omega$-chain end $\left.-\mathrm{CH}_{2} \mathrm{CH}\left(\mathrm{C}_{5} \mathrm{H}_{11}\right) \mathrm{OH}\right), 2.40-2.17\left(\mathrm{~m},-(\mathrm{C}=\mathrm{O}) \mathrm{CH}_{2} \mathrm{CH}_{2}-\right)$, 1.72-1.41 (m, $\left.-(\mathrm{C}=\mathrm{O}) \mathrm{CH}_{2} \mathrm{CH}_{2} \mathrm{CH}_{2} \mathrm{CH}\left(\mathrm{CH}_{2}\left(\mathrm{CH}_{2}\right)_{3} \mathrm{CH}_{3}\right) \mathrm{O}^{-}\right)$, $1.38-1.14$ (br, $\left.-\mathrm{CH}\left(\mathrm{CH}_{2}\left(\mathrm{CH}_{2}\right)_{3} \mathrm{CH}_{3}\right) \mathrm{O}^{-}\right), 0.88$ $\left(\mathrm{t}, J=6.6 \mathrm{~Hz},-\mathrm{CH}_{3}\right)$. 


\section{Synthesis of HO-PDL 22k-OH}

The Method A was used for the polymerization of $\delta$-DL (10.0 g, $58.7 \mathrm{mmol})$ with 1,4-benzenedimethanol (49 $\mathrm{mg}, 0.35 \mathrm{mmol}$ ) and DPP (88 $\mathrm{mg}, 0.35 \mathrm{mmol})$ to give $\mathrm{HO}-\mathrm{PDL}_{22 \mathrm{k}}-\mathrm{OH}$ as a colorless viscous liquid $(7.26 \mathrm{~g})$.

Yield: $72.2 \%$.

$M_{\mathrm{n}, \mathrm{NMR}}=21,600 \mathrm{~g} \mathrm{~mol}^{-1}, M_{\mathrm{n}, \mathrm{SEC}}=24,700 \mathrm{~g} \mathrm{~mol}^{-1}(\mathrm{THF}), Ð=1.07(\mathrm{THF})$.

${ }^{1} \mathrm{H}-\mathrm{NMR}\left(400 \mathrm{MHz}, \mathrm{CDCl}_{3}\right): \delta$ (ppm) 7.34 (s, aromatic), 5.10 (s, - $\mathrm{Ph}-\mathrm{CH}_{2} \mathrm{O}-$ ), 4.87 (quin, main chain $\left.{ }_{-}^{-} \mathrm{CH}_{2} \mathrm{CH}\left(\mathrm{C}_{5} \mathrm{H}_{11}\right) \mathrm{O}^{-}\right)$, 3.66-3.55 (br, $\omega$-chain end $\left.-\mathrm{CH}_{2} \mathrm{CH}\left(\mathrm{C}_{5} \mathrm{H}_{11}\right) \mathrm{OH}\right), 2.48-2.16\left(\mathrm{~m},-(\mathrm{C}=\mathrm{O}) \mathrm{CH}_{2} \mathrm{CH}_{2}^{-}\right)$, 1.85-1.39 (m, $\left.-(\mathrm{C}=\mathrm{O}) \mathrm{CH}_{2} \mathrm{CH}_{2} \mathrm{CH}_{2} \mathrm{CH}\left(\mathrm{CH}_{2}\left(\mathrm{CH}_{2}\right)_{3} \mathrm{CH}_{3}\right) \mathrm{O}^{-}\right)$, $1.37-1.14$ (br, $\left.-\mathrm{CH}\left(\mathrm{CH}_{2}\left(\mathrm{CH}_{2}\right)_{3} \mathrm{CH}_{3}\right) \mathrm{O}^{-}\right), 0.87$ (t, $\left.J=6.8 \mathrm{~Hz},-\mathrm{CH}_{3}\right)$. 
Scheme S7. Synthesis of $\mathrm{N}_{3}-\mathrm{PDL}-\mathrm{N}_{3}$

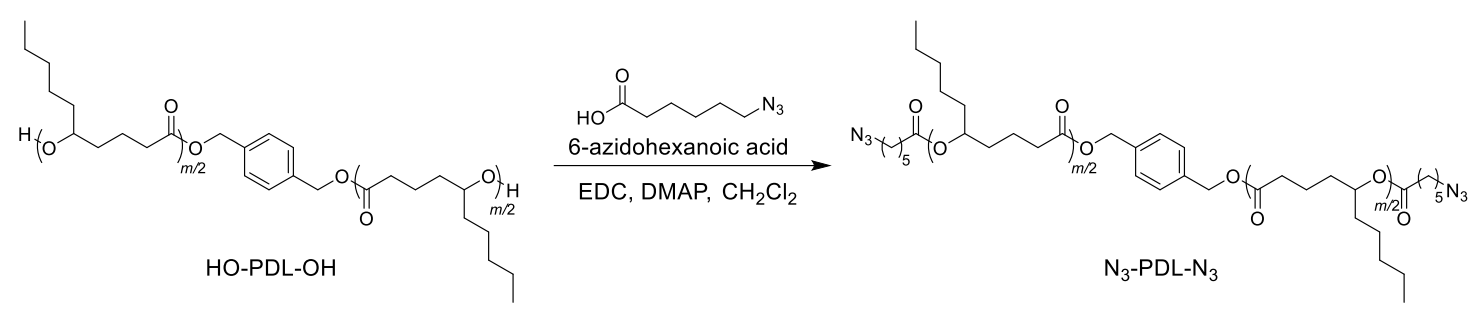

\section{Synthesis of $\mathrm{N}_{3}-\mathrm{PDL}_{6 \mathrm{k}}-\mathrm{N}_{3}$}

A typical end azido-functionalization procedure is as follows (Method B). Prior to the reaction, a trace amount of $\mathrm{MeOH}$ in the $\mathrm{HO}-\mathrm{PDL}_{6 \mathrm{k}}-\mathrm{OH}$ was removed completely by coevaporation with dry toluene. HO-PDL $6 \mathrm{k}-\mathrm{OH}(7.00 \mathrm{~g}, 1.25 \mathrm{mmol})$, 6-azidohexanoic acid (0.787 g, $5.01 \mathrm{mmol}), \mathrm{EDC} \cdot \mathrm{HCl}(1.20 \mathrm{~g}, 6.26$ mmol), DMAP $(0.765 \mathrm{~g}, 6.26 \mathrm{mmol})$, and dry $\mathrm{CH}_{2} \mathrm{Cl}_{2}(70 \mathrm{~mL})$ were mixed in round-bottomed flask. After stirring for $96 \mathrm{~h}$ at room temperature, the mixture was purified in the same way as described in Method A to give $\mathrm{N}_{3}-\mathrm{PDL}_{6 \mathrm{k}}-\mathrm{N}_{3}$ as a colorless viscous liquid (6.38 g). Yield: $86.3 \%$.

$M_{\mathrm{n}, \mathrm{NMR}}=5,900 \mathrm{~g} \mathrm{~mol}^{-1}, M_{\mathrm{n}, \mathrm{SEC}}=9,160 \mathrm{~g} \mathrm{~mol}^{-1}(\mathrm{THF}), Ð=1.07(\mathrm{THF})$.

${ }^{1} \mathrm{H}-\mathrm{NMR}\left(400 \mathrm{MHz}, \mathrm{CDCl}_{3}\right): \delta(\mathrm{ppm}) 7.34$ (s, aromatic), $5.10\left(\mathrm{~s},-\mathrm{Ph}-\mathrm{CH}_{2} \mathrm{O}-\right.$ ), 4.94-4.80 (br, main chain $\left.-\mathrm{CH}_{2} \mathrm{CH}\left(\mathrm{C}_{5} \mathrm{H}_{11}\right) \mathrm{O}^{-}\right), 3.27\left(\mathrm{t}, J=6.9 \mathrm{~Hz},-\mathrm{CH}_{2} \mathrm{~N}_{3}\right), 2.39-2.22\left(\mathrm{~m},-(\mathrm{C}=\mathrm{O}) \mathrm{CH}_{2} \mathrm{CH}_{2}-\right)$, 1.73-1.38 (m, $\left.-(\mathrm{C}=\mathrm{O}) \mathrm{CH}_{2} \mathrm{CH}_{2} \mathrm{CH}_{2} \mathrm{CH}\left(\mathrm{CH}_{2}\left(\mathrm{CH}_{2}\right)_{3} \mathrm{CH}_{3}\right) \mathrm{O}-, \quad-\mathrm{CH}_{2} \mathrm{CH}_{2} \mathrm{CH}_{2} \mathrm{CH}_{2}-\mathrm{N}_{3}\right), \quad 1.38-1.16 \quad$ (br, $\left.-\mathrm{CH}\left(\mathrm{CH}_{2}\left(\mathrm{CH}_{2}\right)_{3} \mathrm{CH}_{3}\right) \mathrm{O}^{-},-\mathrm{CH}_{2}\left(\mathrm{CH}_{2}\right)_{2}-\mathrm{N}_{3}\right), 0.95-0.78\left(\mathrm{~m},-\mathrm{CH}_{3}\right)$. 


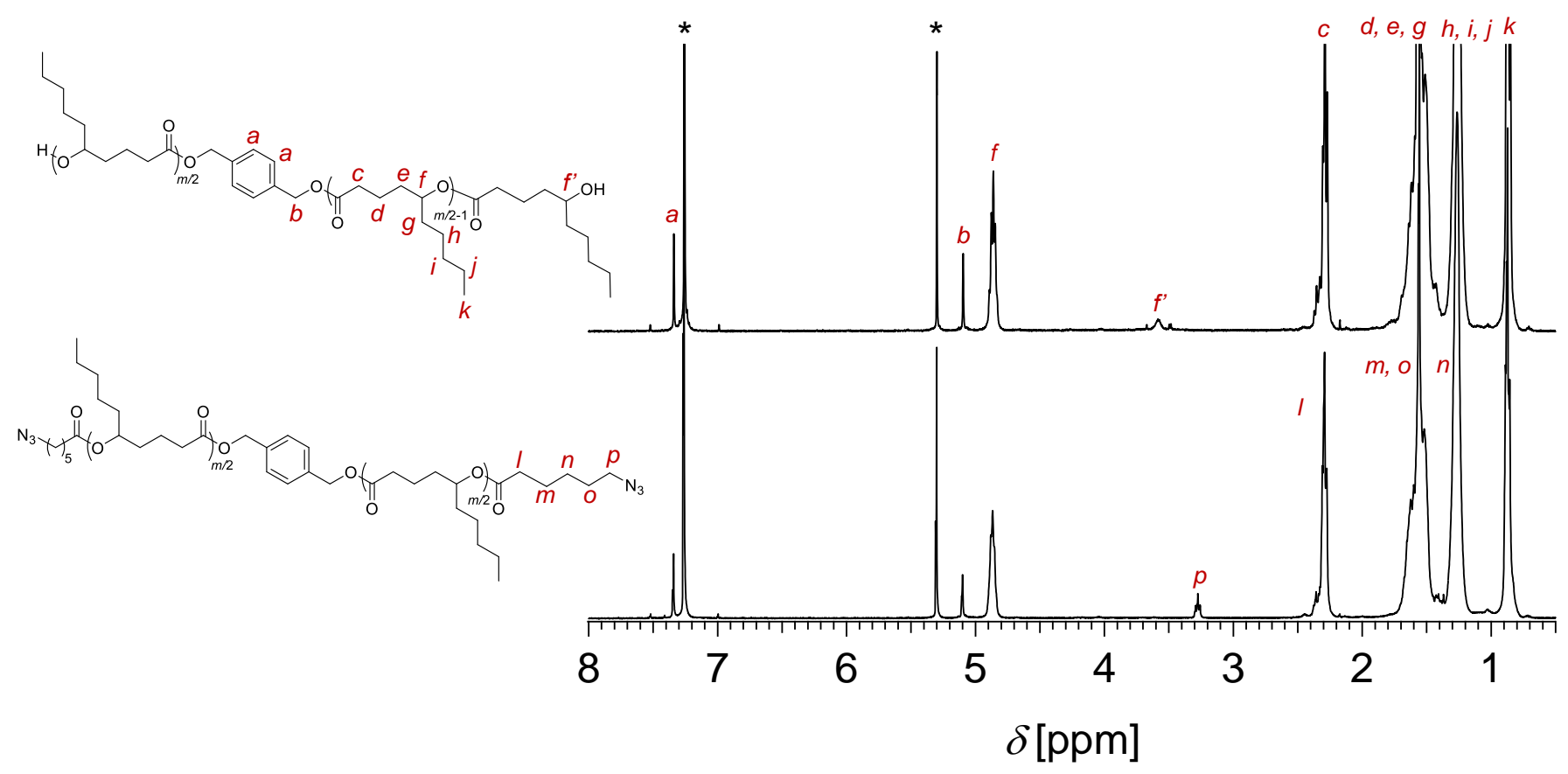

Figure S6. ${ }^{1} \mathrm{H}$ NMR spectra of $\mathrm{HO}-\mathrm{PDL}_{6 \mathrm{k}}-\mathrm{OH}$ (upper) and $\mathrm{N}_{3}-\mathrm{PDL}_{6 \mathrm{k}}-\mathrm{N}_{3}$ (lower) $\left(400 \mathrm{MHz}, \mathrm{CDCl}_{3}\right)$. 


\section{Synthesis of $\mathbf{N}_{3}-P_{10 k}-N_{3}$}

The Method B was used for the reaction of $\mathrm{HO}-\mathrm{PDL}_{10 \mathrm{k}}-\mathrm{OH}(6.00 \mathrm{~g}, 600 \mu \mathrm{mol})$ and 6-azidohexanoic acid $(0.377 \mathrm{~g}, 2.40 \mathrm{mmol})$ in dry $\mathrm{CH}_{2} \mathrm{Cl}_{2}(60 \mathrm{~mL})$ with $\mathrm{EDC} \cdot \mathrm{HCl}(0.575 \mathrm{~g}, 3.00 \mathrm{mmol})$ and DMAP $(0.367 \mathrm{~g}$, $3.00 \mathrm{mmol}$ ) to give $\mathrm{N}_{3}-\mathrm{PDL}_{10 \mathrm{k}}-\mathrm{N}_{3}$ as a colorless viscous liquid (5.94 g). Yield: $96.0 \%$.

$M_{\mathrm{n}, \mathrm{NMR}}=10,300 \mathrm{~g} \mathrm{~mol}^{-1}, M_{\mathrm{n}, \mathrm{SEC}}=14,200 \mathrm{~g} \mathrm{~mol}^{-1}(\mathrm{THF}), Ð=1.06(\mathrm{THF})$.

${ }^{1} \mathrm{H}-\mathrm{NMR}\left(400 \mathrm{MHz}, \mathrm{CDCl}_{3}\right.$ ): $\delta$ (ppm) 7.34 (s, aromatic), 5.10 (s, $-\mathrm{Ph}-\mathrm{CH}_{2} \mathrm{O}-$ ), 4.87 (quin , main chain, $J=$ $\left.5.8 \mathrm{~Hz}-\mathrm{CH}_{2} \mathrm{CH}\left(\mathrm{C}_{5} \mathrm{H}_{11}\right) \mathrm{O}^{-}\right), 3.28\left(\mathrm{t}, J=6.9 \mathrm{~Hz},-\mathrm{CH}_{2} \mathrm{~N}_{3}\right), 2.39-2.22\left(\mathrm{~m},-(\mathrm{C}=\mathrm{O}) \mathrm{CH}_{2} \mathrm{CH}_{2}-\right), 1.73-1.38(\mathrm{~m}$, $\left.-(\mathrm{C}=\mathrm{O}) \mathrm{CH}_{2} \mathrm{CH}_{2} \mathrm{CH}_{2} \mathrm{CH}\left(\mathrm{CH}_{2}\left(\mathrm{CH}_{2}\right)_{3} \mathrm{CH}_{3}\right) \mathrm{O}-, \quad-\mathrm{CH}_{2} \mathrm{CH}_{2} \mathrm{CH}_{2} \mathrm{CH}_{2}-\mathrm{N}_{3}\right), \quad 1.38-1.16 \quad$ (br, $\left.-\mathrm{CH}\left(\mathrm{CH}_{2}\left(\mathrm{CH}_{2}\right)_{3} \mathrm{CH}_{3}\right) \mathrm{O}-,-\mathrm{CH}_{2}\left(\mathrm{CH}_{2}\right)_{2}-\mathrm{N}_{3}\right), 0.88\left(\mathrm{t}, J=6.6 \mathrm{~Hz},-\mathrm{CH}_{3}\right)$.

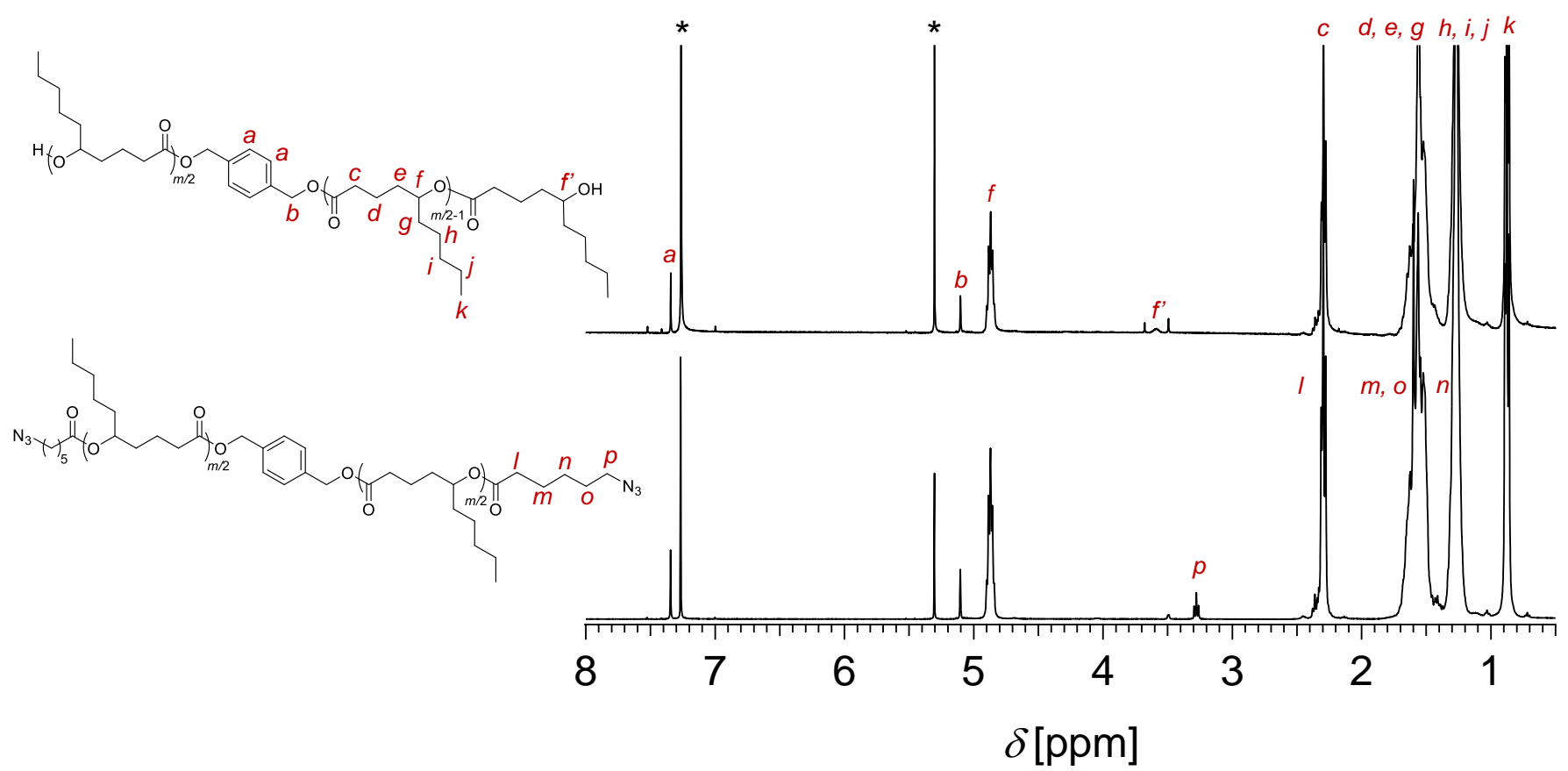

Figure S7. ${ }^{1} \mathrm{H}$ NMR spectra of $\mathrm{HO}-\mathrm{PDL}_{10 \mathrm{k}}-\mathrm{OH}$ (upper) and $\mathrm{N}_{3}-\mathrm{PDL}_{10 \mathrm{k}}-\mathrm{N}_{3}$ (lower) $\left(400 \mathrm{MHz}, \mathrm{CDCl}_{3}\right)$. 


\section{Synthesis of $\mathbf{N}_{3}-P_{22}-N_{3}$}

The Method B was used for the reaction of $\mathrm{HO}-\mathrm{PDL}_{22 \mathrm{k}}-\mathrm{OH}(7.00 \mathrm{~g}, 324 \mu \mathrm{mol})$ and 6-azidohexanoic acid $(0.204 \mathrm{~g}, 1.30 \mathrm{mmol})$ in dry $\mathrm{CH}_{2} \mathrm{Cl}_{2}(70 \mathrm{~mL})$ with $\mathrm{EDC} \cdot \mathrm{HCl}(0.311 \mathrm{~g}, 1.62 \mathrm{mmol})$ and DMAP $(198 \mathrm{mg}, 1.62$ mmol) to give $\mathrm{N}_{3}-\mathrm{PDL}_{22 \mathrm{k}}-\mathrm{N}_{3}$ as a colorless viscous liquid $(7.75 \mathrm{~g})$. Yield: $>99 \%$.

$M_{\mathrm{n}, \mathrm{NMR}}=21,900 \mathrm{~g} \mathrm{~mol}^{-1}, M_{\mathrm{n}, \mathrm{SEC}}=24,900 \mathrm{~g} \mathrm{~mol}^{-1}(\mathrm{THF}), \oslash=1.09(\mathrm{THF})$.

${ }^{1} \mathrm{H}-\mathrm{NMR}\left(400 \mathrm{MHz}, \mathrm{CDCl}_{3}\right.$ ): $\delta$ (ppm) 7.34 (s, aromatic), 5.10 (s, $-\mathrm{Ph}-\mathrm{CH}_{2} \mathrm{O}-$ ), 4.87 (quin , main chain, $J=$ $\left.6.0 \mathrm{~Hz},-\mathrm{CH}_{2} \mathrm{CH}\left(\mathrm{C}_{5} \mathrm{H}_{11}\right) \mathrm{O}-\right), 3.28\left(\mathrm{t}, J=6.9 \mathrm{~Hz},-\mathrm{CH}_{2} \mathrm{~N}_{3}\right), 2.39-2.23\left(\mathrm{~m},-(\mathrm{C}=\mathrm{O}) \mathrm{CH}_{2} \mathrm{CH}_{2}-\right), 1.73-1.39(\mathrm{~m}$, $\left.-(\mathrm{C}=\mathrm{O}) \mathrm{CH}_{2} \mathrm{CH}_{2} \mathrm{CH}_{2} \mathrm{CH}\left(\mathrm{CH}_{2}\left(\mathrm{CH}_{2}\right)_{3} \mathrm{CH}_{3}\right) \mathrm{O}-, \quad-\mathrm{CH}_{2} \mathrm{CH}_{2} \mathrm{CH}_{2} \mathrm{CH}_{2}-\mathrm{N}_{3}\right), \quad$ 1.39-1.16 (br, $\left.-\mathrm{CH}\left(\mathrm{CH}_{2}\left(\mathrm{CH}_{2}\right)_{3} \mathrm{CH}_{3}\right) \mathrm{O}-,-\mathrm{CH}_{2}\left(\mathrm{CH}_{2}\right)_{2}-\mathrm{N}_{3}\right), 0.88\left(\mathrm{t}, J=6.6 \mathrm{~Hz},-\mathrm{CH}_{3}\right)$.

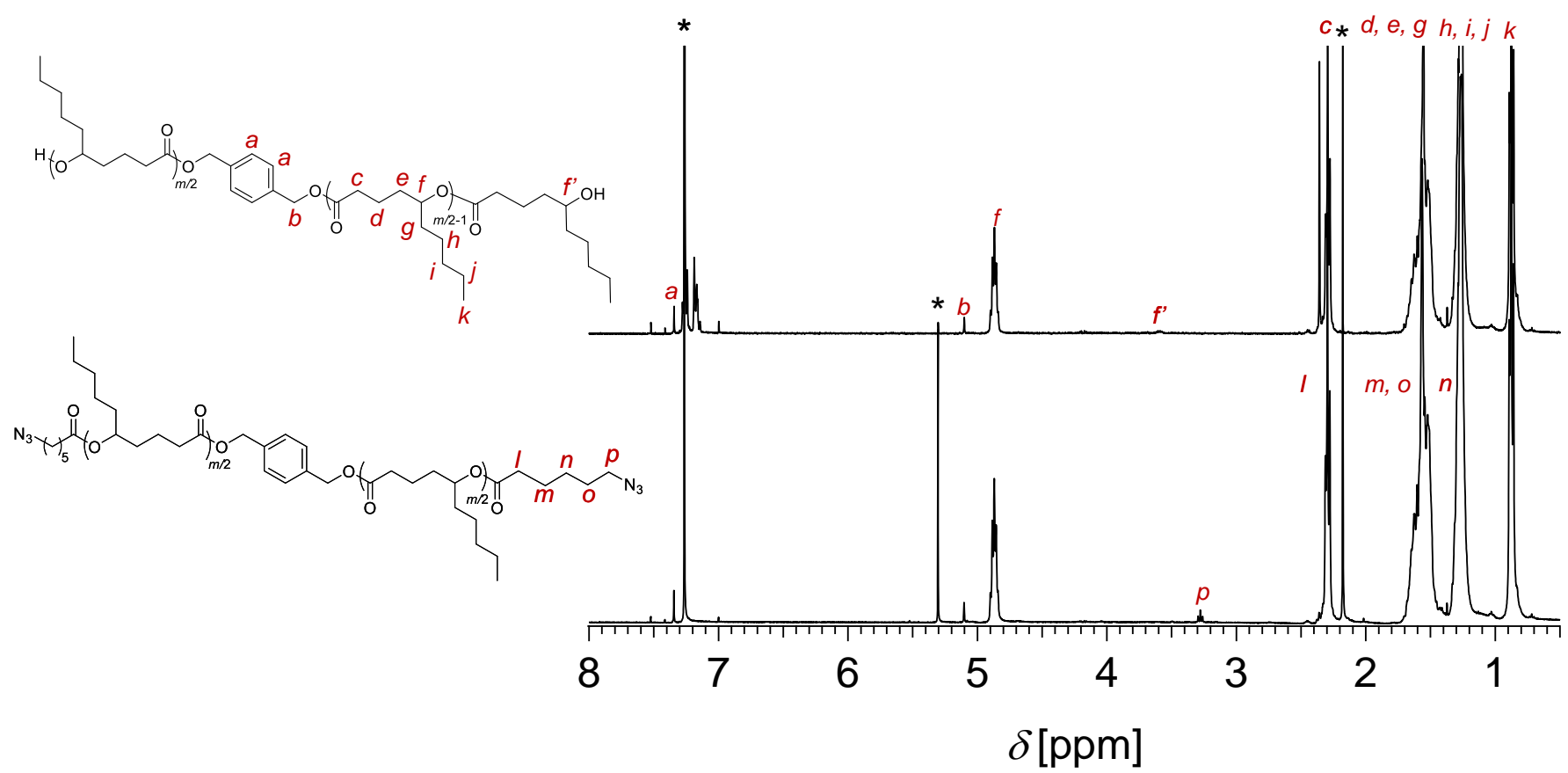

Figure S8. ${ }^{1} \mathrm{H}$ NMR spectra of $\mathrm{HO}-\mathrm{PDL}_{22 \mathrm{k}}-\mathrm{OH}$ (upper) and $\mathrm{N}_{3}-\mathrm{PDL}_{22 \mathrm{k}}-\mathrm{N}_{3}$ (lower) (400 MHz, $\left.\mathrm{CDCl}_{3}\right)$. 
Synthesis of ABA-type BCP consisting of cellooligosaccharide acetate and poly $(\delta$-decanolactone $)$ $\left(\operatorname{AcCel}_{n}-b-\mathrm{PDL}-b-\mathrm{AcCel}_{n}\right)$ via click reaction

Scheme S8. Synthesis of $\mathrm{AcCel}_{n}-b$-PDL- $b-\mathrm{AcCel}_{n}$
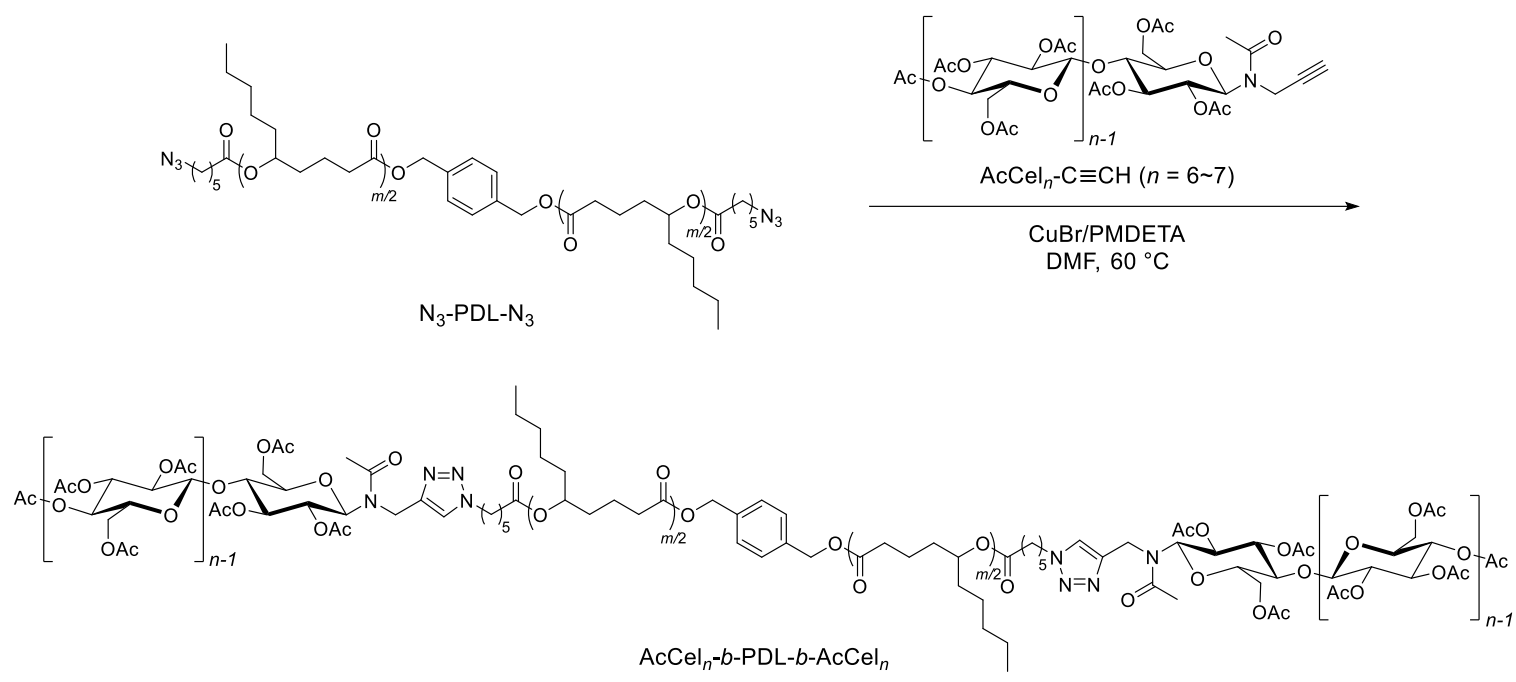

\section{Synthesis of $\mathrm{AcCel}_{n}-b-\mathrm{PDL}_{6 \mathrm{k}}-b-\mathrm{AcCel}_{n}$}

A typical click reaction procedure is as follows (Method C). A degassed solution of $\mathrm{N}_{3}-\mathrm{PDL}_{6 \mathrm{k}}-\mathrm{N}_{3}$ $\left(M_{\mathrm{n}, \mathrm{NMR}}=5,900 \mathrm{~g} \mathrm{~mol}^{-1}, 1.00 \mathrm{~g}, 169 \mu \mathrm{mol}\right)$ and PMDETA $(124 \mu \mathrm{L}, 593 \mu \mathrm{mol})$ in DMF $(10 \mathrm{~mL})$ was transferred to a Schlenk tube in which $\mathrm{AcCel}_{n}-\mathrm{C} \equiv \mathrm{CH}(925 \mathrm{mg}, 441 \mu \mathrm{mol})$ and $\mathrm{CuBr}(85 \mathrm{mg}, 0.59 \mathrm{mmol})$ were placed. The mixture was stirred for $55 \mathrm{~h}$ at $65{ }^{\circ} \mathrm{C}$ under an argon atmosphere. After cooling to room temperature, Dowex ${ }^{\circledR} 50 \mathrm{WX} 2$ and a few drops of water were added to remove $\mathrm{Cu}$ catalyst. The polymer was purified by the preparative $\mathrm{SEC}$ using $\mathrm{CHCl}_{3}$ to remove unreacted $\mathrm{AcCel}_{n}-\mathrm{C} \equiv \mathrm{CH}$, and the resulted solution was concentrated and dried under vacuum to give $\mathrm{AcCel}_{n}-b-\mathrm{PDL}_{6 \mathrm{k}}-b-\mathrm{AcCel}_{n}$ as a yellow elastic material (1.16 g). Yield: $66.2 \%$.

$M_{\mathrm{n}, \mathrm{SEC}}=14,100 \mathrm{~g} \mathrm{~mol}^{-1}(\mathrm{THF}), Ð=1.04(\mathrm{THF}), M_{\mathrm{n}, \mathrm{total}}=10,100 \mathrm{~g} \mathrm{~mol}^{-1}$.

${ }^{1} \mathrm{H}$ NMR (400 MHz, $\left.\mathrm{CDCl}_{3}\right): \delta(\mathrm{ppm})$ 7.52, 7.41 (2x s, rotamers, triazole methine), 7.34 (s, aromatic), 5.97-5.90 (br, $-\mathrm{CH}(\mathrm{NAc})-)$, 5.27-3.45 (m, $-\mathrm{Ph}-\mathrm{CH}_{2} \mathrm{O}-,-\mathrm{CH}_{2} \mathrm{CH}\left(\mathrm{C}_{5} \mathrm{H}_{11}\right) \mathrm{O}-,-\mathrm{CH}_{2} \mathrm{CH}_{2}-$ triazole-, $\mathrm{H}-1,2$, $\left.-3,-4,-5,-6^{\mathrm{AcCel} n},-\mathrm{N}(\mathrm{Ac}) \mathrm{CH}_{2}-\right), 2.45-1.83\left(\mathrm{~m},-(\mathrm{C}=\mathrm{O}) \mathrm{CH}_{2} \mathrm{CH}_{2}-, \mathrm{CH}_{3}\right.$ in $\left.\mathrm{Ac}^{\mathrm{AcCel} n},-\mathrm{N}(\mathrm{C}=\mathrm{O}) \mathrm{CH}_{3}\right), 1.77-1.42$, 
(m, $\quad-(\mathrm{C}=\mathrm{O}) \mathrm{CH}_{2}\left(\mathrm{CH}_{2}\right)_{2} \mathrm{CH}\left(\mathrm{CH}_{2}\left(\mathrm{CH}_{2}\right)_{3} \mathrm{CH}_{3}\right) \mathrm{O}^{-}, \quad-\mathrm{CH}_{2} \mathrm{CH}_{2} \mathrm{CH}_{2} \mathrm{CH}_{2}-$ triazole- $), \quad 1.42-0.99, \quad$ (m, $-\mathrm{CH}\left(\mathrm{CH}_{2}\left(\mathrm{CH}_{2}\right)_{3} \mathrm{CH}_{3}\right) \mathrm{O}-,-\mathrm{CH}_{2}\left(\mathrm{CH}_{2}\right)_{2}-$ triazole- $), 0.88\left(\mathrm{t}, J=6.6 \mathrm{~Hz},-\mathrm{CH}_{3}\right)$ 


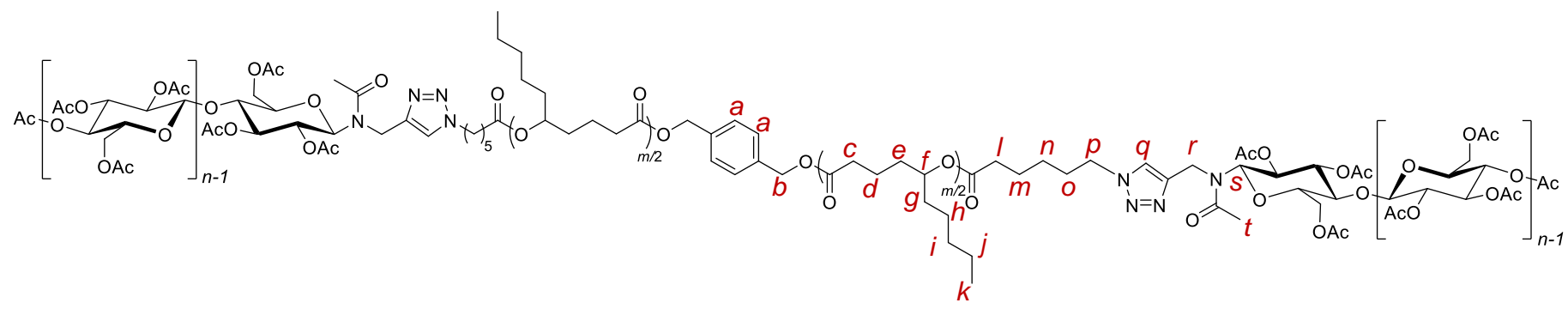

(a)

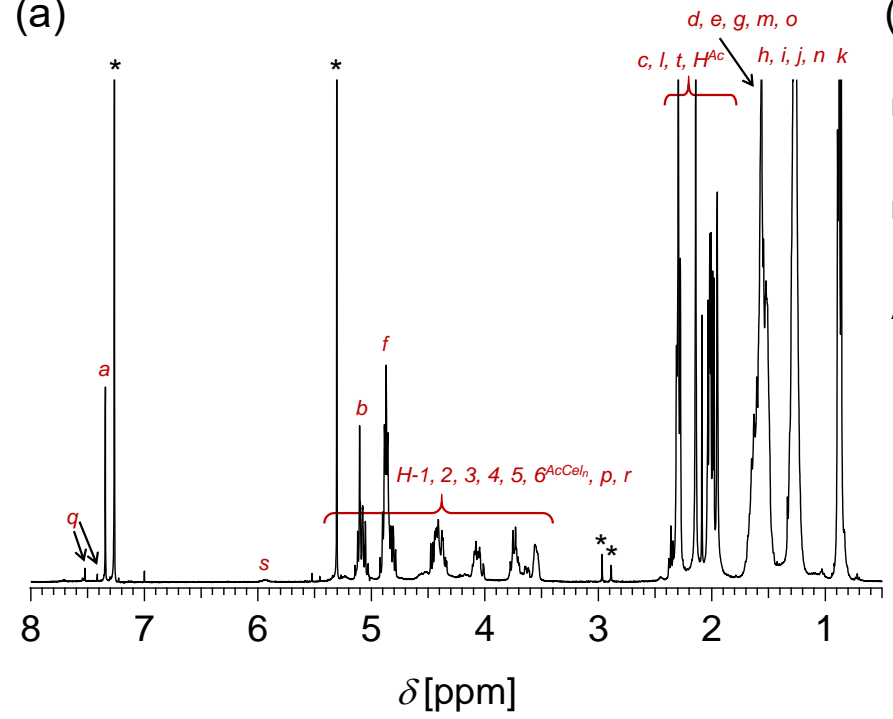

(b)

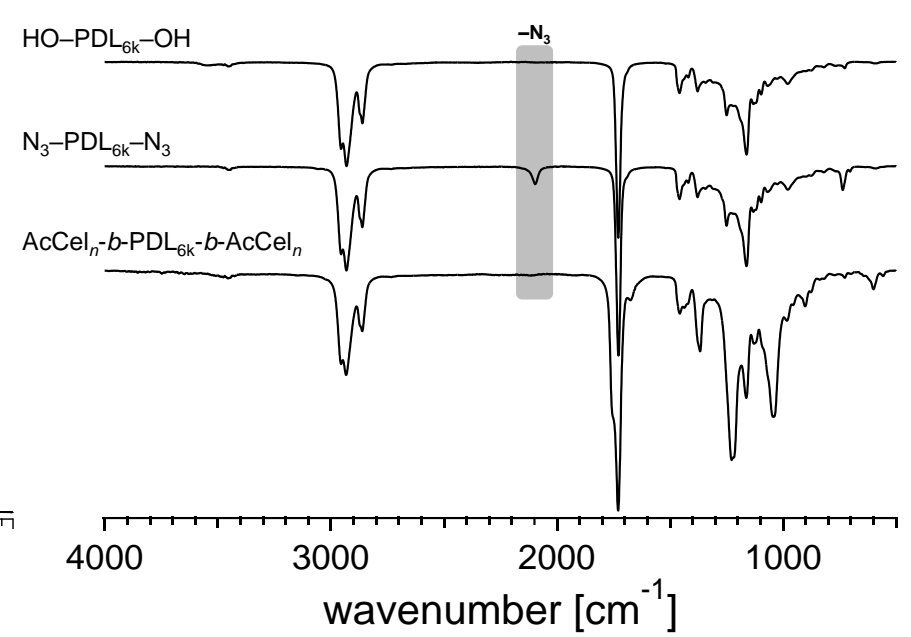

Figure S9. Characterization of $\mathrm{AcCel}_{n}-b-\mathrm{PDL}_{6 \mathrm{k}}-b-\mathrm{AcCel}_{n}$. (a) ${ }^{1} \mathrm{H} \mathrm{NMR}$ spectrum $\left(\mathrm{CDCl}_{3}, 400 \mathrm{MHz}\right)$. (b) FTIR spectra of $\mathrm{HO}-\mathrm{PDL}_{6 \mathrm{k}}-\mathrm{OH}$ (upper), $\mathrm{N}_{3}-\mathrm{PDL}_{6 \mathrm{k}}-\mathrm{N}_{3}$ (middle), and $\mathrm{AcCel}_{n}-b-\mathrm{PDL}_{6 \mathrm{k}}-b-\mathrm{AcCel}_{n}$ (lower). 


\section{Synthesis of $\mathrm{AcCel}_{n}-b-\mathrm{PDL}_{10 \mathrm{k}}-b-\mathrm{AcCel}_{n}$}

The Method C was used for the click reaction of $\mathrm{N}_{3}-\mathrm{PDL}_{10 \mathrm{k}}-\mathrm{N}_{3}\left(M_{\mathrm{n}, \mathrm{NMR}}=10,300 \mathrm{~g} \mathrm{~mol}^{-1}, 1.00 \mathrm{~g}\right.$, $97.0 \mu \mathrm{mol})$ and $\mathrm{AcCel}_{n}-\mathrm{C} \equiv \mathrm{CH}(530 \mathrm{mg}, 252 \mu \mathrm{mol})$ in DMF $(10 \mathrm{~mL})$ with $\mathrm{CuBr}(39 \mathrm{mg}, 0.27 \mathrm{mmol})$ and PMDETA $(57 \mu \mathrm{L}, 0.27 \mathrm{mmol})$ to synthesize $\mathrm{AcCel}_{n}-b-\mathrm{PDL}_{10 \mathrm{k}}-b-\mathrm{AcCel}_{n}$. The crude product was purified by preparative SEC to give $\mathrm{AcMal}_{n}-b-\mathrm{PDL}_{10 \mathrm{k}}-b-\mathrm{AcMal}_{n}$ as a yellow elastic material (1.13 g). Yield: $80.3 \%$.

$M_{\mathrm{n}, \mathrm{SEC}}=19,200 \mathrm{~g} \mathrm{~mol}^{-1}(\mathrm{THF}), Ð=1.04(\mathrm{THF}), M_{\mathrm{n}, \mathrm{total}}=14,500 \mathrm{~g} \mathrm{~mol}^{-1}$.

${ }^{1} \mathrm{H}$ NMR (400 MHz, $\left.\mathrm{CDCl}_{3}\right): \delta(\mathrm{ppm})$ 7.52, 7.41 (2x s, rotamers, triazole methine), 7.34 (s, aromatic), 5.96-5.90 (br, $-\mathrm{CH}(\mathrm{NAc})-), 5.23-3.47\left(\mathrm{~m},-\mathrm{Ph}-\mathrm{CH}_{2} \mathrm{O}-,-\mathrm{CH}_{2} \mathrm{CH}\left(\mathrm{C}_{5} \mathrm{H}_{11}\right) \mathrm{O}^{-},-\mathrm{CH}_{2} \mathrm{CH}_{2}-\right.$ triazole-, $\mathrm{H}-1,2$, $\left.-3,-4,-5,-6^{\mathrm{AcCel} n},-\mathrm{N}(\mathrm{Ac}) \mathrm{CH}_{2}-\right), 2.49-1.87\left(\mathrm{~m},-(\mathrm{C}=\mathrm{O}) \mathrm{CH}_{2} \mathrm{CH}_{2}-, \mathrm{CH}_{3}\right.$ in $\left.\mathrm{Ac}^{\mathrm{AcCel} n},-\mathrm{N}(\mathrm{C}=\mathrm{O}) \mathrm{CH}_{3}\right), 1.76-$ 1.41, (m, $-(\mathrm{C}=\mathrm{O}) \mathrm{CH}_{2} \mathrm{CH}_{2} \mathrm{CH}_{2} \mathrm{CH}\left(\mathrm{CH}_{2}\left(\mathrm{CH}_{2}\right)_{3} \mathrm{CH}_{3}\right) \mathrm{O}-, \quad-\mathrm{CH}_{2} \mathrm{CH}_{2} \mathrm{CH}_{2} \mathrm{CH}_{2}-$ triazole- $), \quad 1.38-1.15$, (m, $-\mathrm{CH}\left(\mathrm{CH}_{2}\left(\mathrm{CH}_{2}\right)_{3} \mathrm{CH}_{3}\right) \mathrm{O}-,-\mathrm{CH}_{2}\left(\mathrm{CH}_{2}\right)_{2}-$ triazole -$), 0.88\left(\mathrm{t}, \mathrm{J}=6.8 \mathrm{~Hz},-\mathrm{CH}_{3}\right)$. 

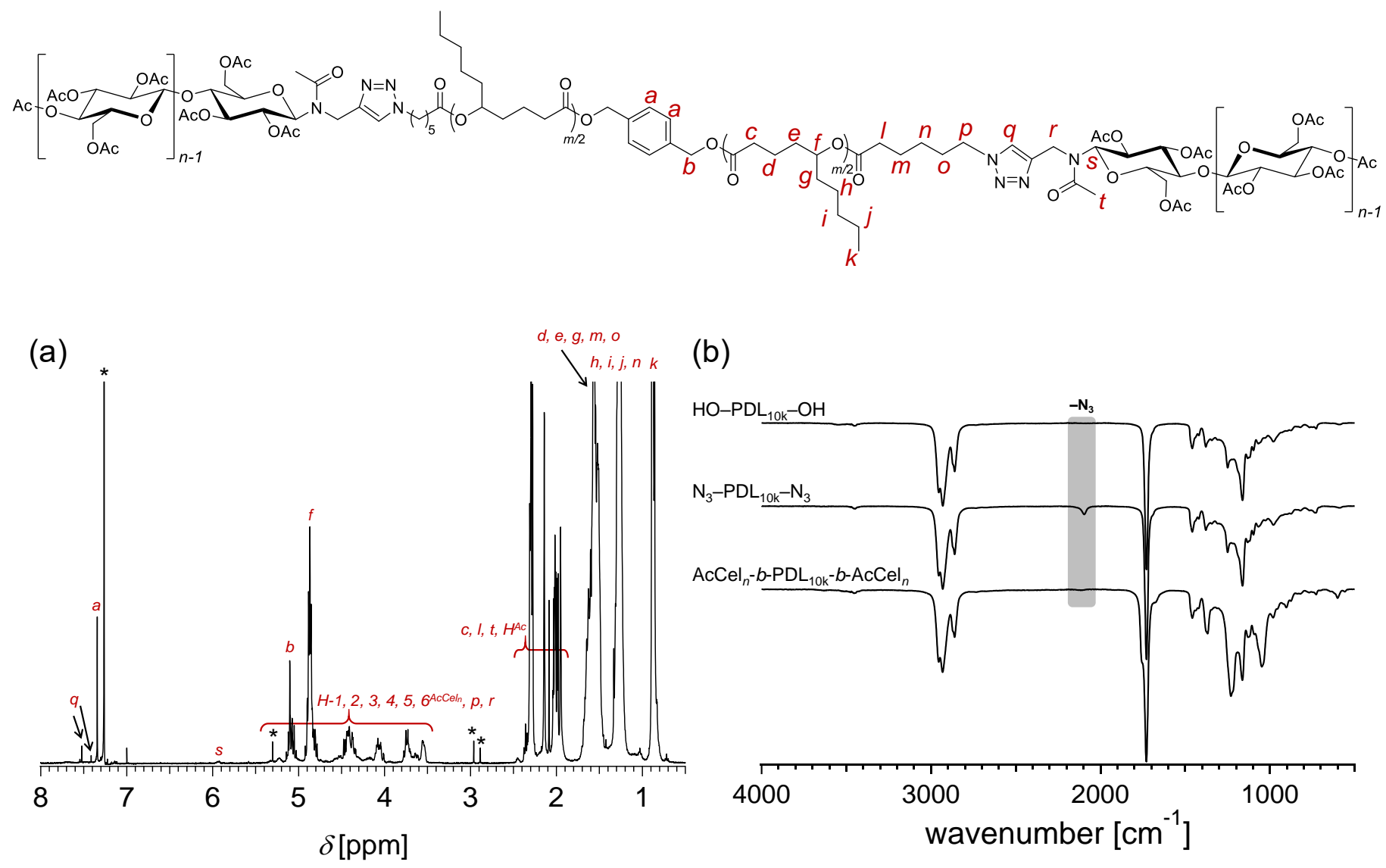

(b)

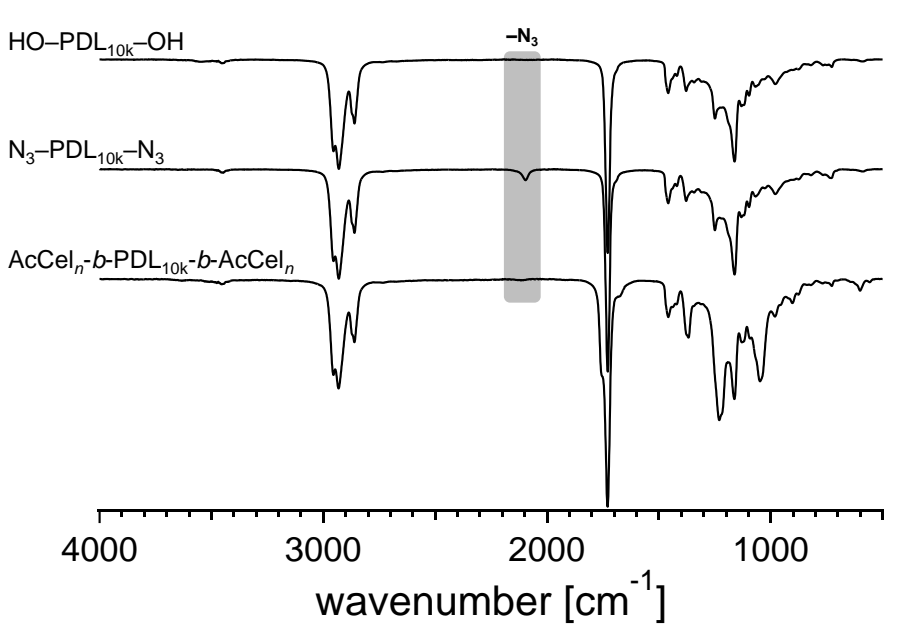

Figure S10. Characterization of $\mathrm{AcCel}_{n}-b-\mathrm{PDL}_{10 \mathrm{k}}-b-\mathrm{AcCel}_{n}$. (a) ${ }^{1} \mathrm{H} \mathrm{NMR}$ spectrum $\left(\mathrm{CDCl}_{3}, 400 \mathrm{MHz}\right)$. (b) FT-IR spectra of $\mathrm{HO}-\mathrm{PDL}_{10 \mathrm{k}}-\mathrm{OH}$ (upper), $\mathrm{N}_{3}-\mathrm{PDL}_{10 \mathrm{k}}-\mathrm{N}_{3}$ (middle), and $\mathrm{AcCel}_{n}-b-\mathrm{PDL}_{10 \mathrm{k}}-b-\mathrm{AcCl}_{n}$ (lower). 


\section{Synthesis of $\operatorname{AcCel}_{n}-b-P_{22 k}-b-A_{c C e l}$}

The Method C was used for the click reaction of $\mathrm{N}_{3}-\mathrm{PDL}_{22 \mathrm{k}}-\mathrm{N}_{3}\left(M_{\mathrm{n}, \mathrm{NMR}}=21,900 \mathrm{~g} \mathrm{~mol}^{-1}, 1.00 \mathrm{~g}\right.$, $46.0 \mu \mathrm{mol})$ and $\mathrm{AcCel}_{n}-\mathrm{C} \equiv \mathrm{CH}(252 \mathrm{mg}, 120 \mu \mathrm{mol})$ in $\mathrm{DMF}(10 \mathrm{~mL})$ with $\mathrm{CuBr}(23 \mathrm{mg}, 0.16 \mathrm{mmol})$ and PMDETA $(34 \mu \mathrm{L}, 0.16 \mathrm{mmol})$. The crude product was purified by preparative SEC to give $\mathrm{AcMal}_{n}-b-\mathrm{PDL}_{22 \mathrm{k}}-$ $b-\mathrm{AcMal}_{n}$ as a yellow elastic material $(537 \mathrm{mg})$. Yield: $45.0 \%$.

$M_{\mathrm{n}, \mathrm{SEC}}=30,800 \mathrm{~g} \mathrm{~mol}^{-1}(\mathrm{THF}), Ð=1.06(\mathrm{THF}), M_{\mathrm{n}, \mathrm{total}}=26,100 \mathrm{~g} \mathrm{~mol}^{-1}$.

${ }^{1} \mathrm{H}$ NMR (400 MHz, $\left.\mathrm{CDCl}_{3}\right): \delta(\mathrm{ppm})$ 7.52, 7.41 (2x s, rotamers, triazole methine), 7.34 (s, aromatic), 5.97-5.90 (br, $-\mathrm{CH}(\mathrm{NAc})-), 5.28-3.47\left(\mathrm{~m},-\mathrm{Ph}-\mathrm{CH}_{2} \mathrm{O}-,-\mathrm{CH}_{2} \mathrm{CH}\left(\mathrm{C}_{5} \mathrm{H}_{11}\right) \mathrm{O}^{-},-\mathrm{CH}_{2} \mathrm{CH}_{2}-\right.$ triazole-, $\mathrm{H}-1,2$,

$\left.-3,-4,-5,-6^{\mathrm{AcCel} n},-\mathrm{N}(\mathrm{Ac}) \mathrm{CH}_{2}-\right), 2.49-1.86\left(\mathrm{~m},-(\mathrm{C}=\mathrm{O}) \mathrm{CH}_{2} \mathrm{CH}_{2}-, \mathrm{CH}_{3}\right.$ in $\left.\mathrm{Ac}^{\mathrm{AcCel} n},-\mathrm{N}(\mathrm{C}=\mathrm{O}) \mathrm{CH}_{3}\right), 1.83-$ $1.39\left(\mathrm{~m}, \quad-(\mathrm{C}=\mathrm{O}) \mathrm{CH}_{2} \mathrm{CH}_{2} \mathrm{CH}_{2} \mathrm{CH}\left(\mathrm{CH}_{2}\left(\mathrm{CH}_{2}\right)_{3} \mathrm{CH}_{3}\right) \mathrm{O}-, \quad-\mathrm{CH}_{2} \mathrm{CH}_{2} \mathrm{CH}_{2} \mathrm{CH}_{2}\right.$-triazole- $), \quad 1.39-0.96, \quad$ (m, $-\mathrm{CH}\left(\mathrm{CH}_{2}\left(\mathrm{CH}_{2}\right)_{3} \mathrm{CH}_{3}\right) \mathrm{O}^{-},-\mathrm{CH}_{2}\left(\mathrm{CH}_{2}\right)_{2}-$ triazole- $), 0.88\left(\mathrm{t}, \mathrm{J}=6.6 \mathrm{~Hz},-\mathrm{CH}_{3}\right)$. 

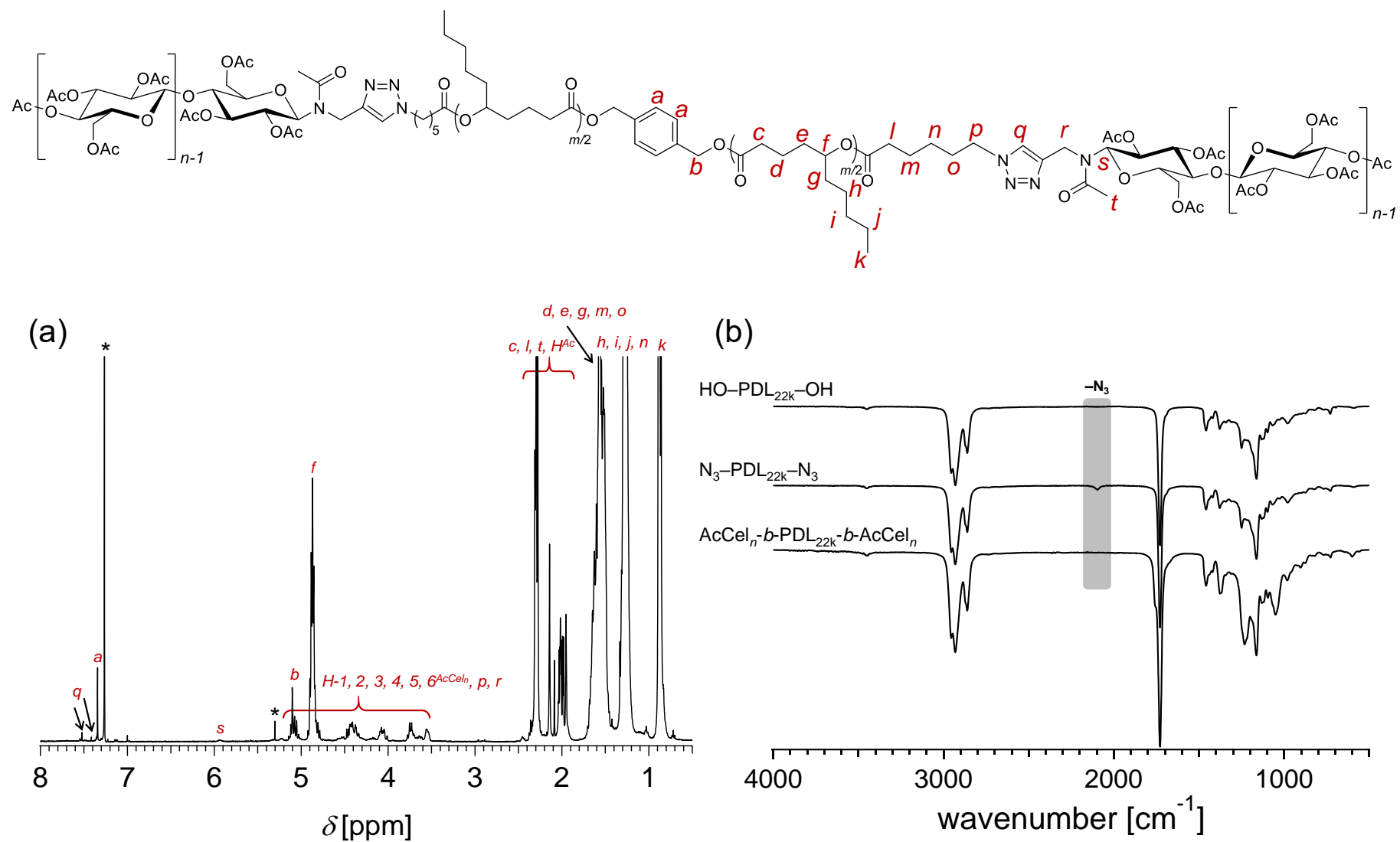

(b)

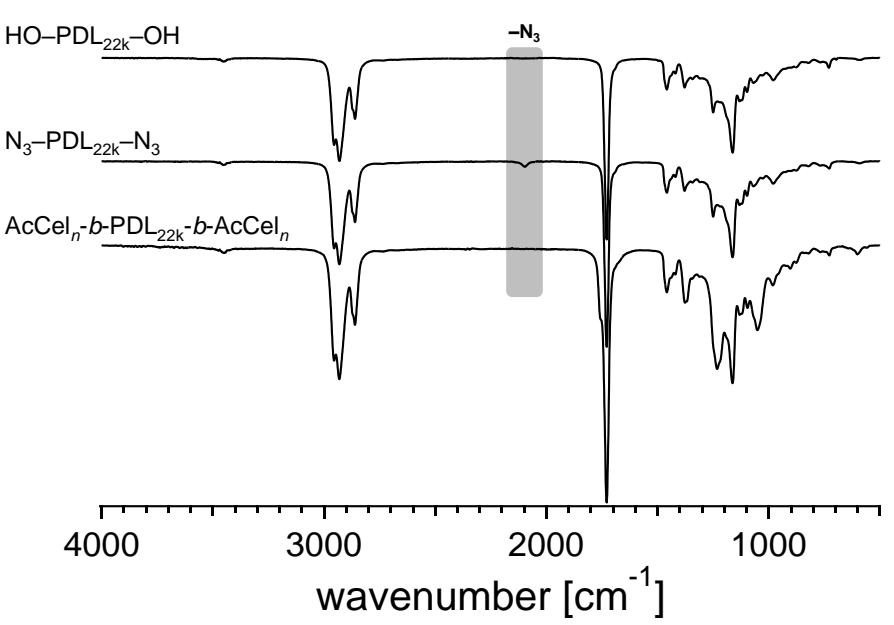

Figure S11. Characterization of $\mathrm{AcCel}_{n}-b-\mathrm{PDL}_{22 \mathrm{k}}-b-\mathrm{AcCel}_{n}$. (a) ${ }^{1} \mathrm{H} \mathrm{NMR}$ spectrum $\left(\mathrm{CDCl}_{3}, 400 \mathrm{MHz}\right)$. (b) FT-IR spectra of $\mathrm{HO}-\mathrm{PDL}_{22 \mathrm{k}}-\mathrm{OH}$ (upper), $\mathrm{N}_{3}-\mathrm{PDL}_{22 \mathrm{k}}-\mathrm{N}_{3}$ (middle), and $\mathrm{AcCel}_{n}-b$-PDL $22 \mathrm{k}-b$-AcCel ${ }_{n}$ (lower). 
Synthesis of ABA-type BCP consisting of maltooligosaccharide acetate and poly $(\delta$-decanolactone $)$

$\left(\mathrm{AcMal}_{n}-b-\mathrm{PDL}-b-\mathrm{AcMal}_{n}\right)$ via click reaction

Scheme S9. Synthesis of $\mathrm{AcMal}_{n}-b-\mathrm{PDL}-b-\mathrm{AcMal}_{n}$
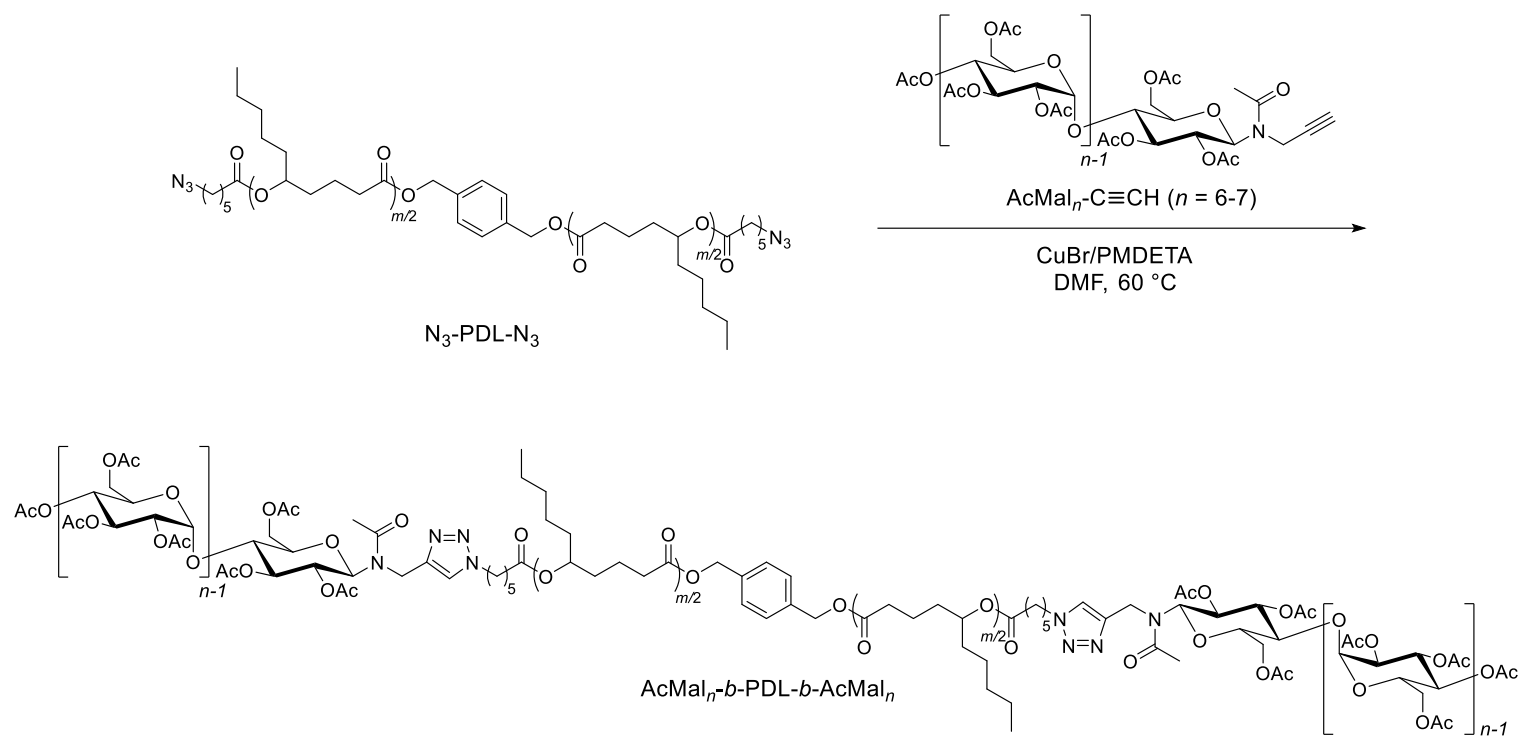

\section{Synthesis of $\mathrm{AcMal}_{n}-b-\mathrm{PDL}_{6 \mathrm{k}}-b-\mathrm{AcMal}_{n}$}

The Method $\mathrm{C}$ was used for the click reaction of $\mathrm{N}_{3}-\mathrm{PDL}_{6 \mathrm{k}}-\mathrm{N}_{3}\left(M_{\mathrm{n}, \mathrm{NMR}}=5,900 \mathrm{~g} \mathrm{~mol}^{-1}, 1.00 \mathrm{~g}, 169\right.$ $\mu \mathrm{mol})$ and $\mathrm{AcMal}_{n}-\mathrm{C} \equiv \mathrm{CH}(859 \mathrm{mg}, 441 \mu \mathrm{mol})$ in $\mathrm{DMF}(10 \mathrm{~mL})$ with $\mathrm{CuBr}(85 \mathrm{mg}, 593 \mu \mathrm{mol})$ and PMDETA $(124 \mu \mathrm{L}, 593 \mu \mathrm{mol})$. The crude product was purified by the preparative SEC to give $\mathrm{AcMal}_{n}-b-\mathrm{PDL}_{10 \mathrm{k}}-b$ $\mathrm{AcMal}_{n}$ as a yellow elastic material (1.02 g). Yield: $60.1 \%$.

$M_{\mathrm{n}, \mathrm{SEC}}=12,000 \mathrm{~g} \mathrm{~mol}^{-1}(\mathrm{THF}), \bigoplus=1.04(\mathrm{THF}), M_{\mathrm{n}, \mathrm{total}}=9,800 \mathrm{~g} \mathrm{~mol}^{-1}$.

${ }^{1} \mathrm{H}$ NMR (400 MHz, $\mathrm{CDCl}_{3}$ ): $\delta$ (ppm) 7.52, 7.41 (2x s, rotamers, triazole methine), 7.34 (s, aromatic), 6.06$5.98(\mathrm{br},-\mathrm{CH}(\mathrm{NAc})-), 5.49-3.70\left(\mathrm{~m},-\mathrm{Ph}-\mathrm{CH}_{2} \mathrm{O}-,-\mathrm{CH}_{2} \mathrm{CH}\left(\mathrm{C}_{5} \mathrm{H}_{11}\right) \mathrm{O}-,-\mathrm{CH}_{2} \mathrm{CH}_{2}-\right.$ triazole- $, \mathrm{H}-1,2,-3,-4$, $\left.-5,-6^{\mathrm{AcMal} n},-\mathrm{N}(\mathrm{Ac}) \mathrm{CH}_{2}-\right), 2.49-1.83\left(\mathrm{~m},-(\mathrm{C}=\mathrm{O}) \mathrm{CH}_{2} \mathrm{CH}_{2}-, \mathrm{CH}_{3}\right.$ in $\left.\mathrm{Ac}^{\mathrm{AcMal} n},-\mathrm{N}(\mathrm{C}=\mathrm{O}) \mathrm{CH}_{3}\right), 1.77-1.40,(\mathrm{~m}$, main chain $-\left(\mathrm{CH}_{2}\right)_{2} \mathrm{CH}\left(\mathrm{CH}_{2}\left(\mathrm{CH}_{2}\right)_{3} \mathrm{CH}_{3}\right) \mathrm{O}-, \quad-\mathrm{CH}_{2} \mathrm{CH}_{2} \mathrm{CH}_{2} \mathrm{CH}_{2}$-triazole), $\quad 1.38-0.98 \quad$ (m, $-\mathrm{CH}\left(\mathrm{CH}_{2}\left(\mathrm{CH}_{2}\right)_{3} \mathrm{CH}_{3}\right) \mathrm{O}-,-\mathrm{CH}_{2}\left(\mathrm{CH}_{2}\right)_{2}$-triazole), $0.88\left(\mathrm{t}, J=6.6 \mathrm{~Hz},-\mathrm{CH}_{3}\right)$. 


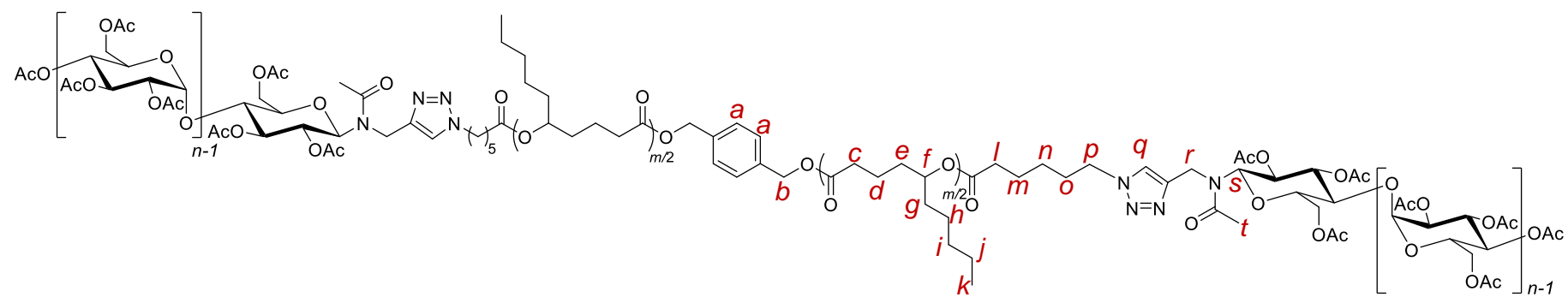

(a)

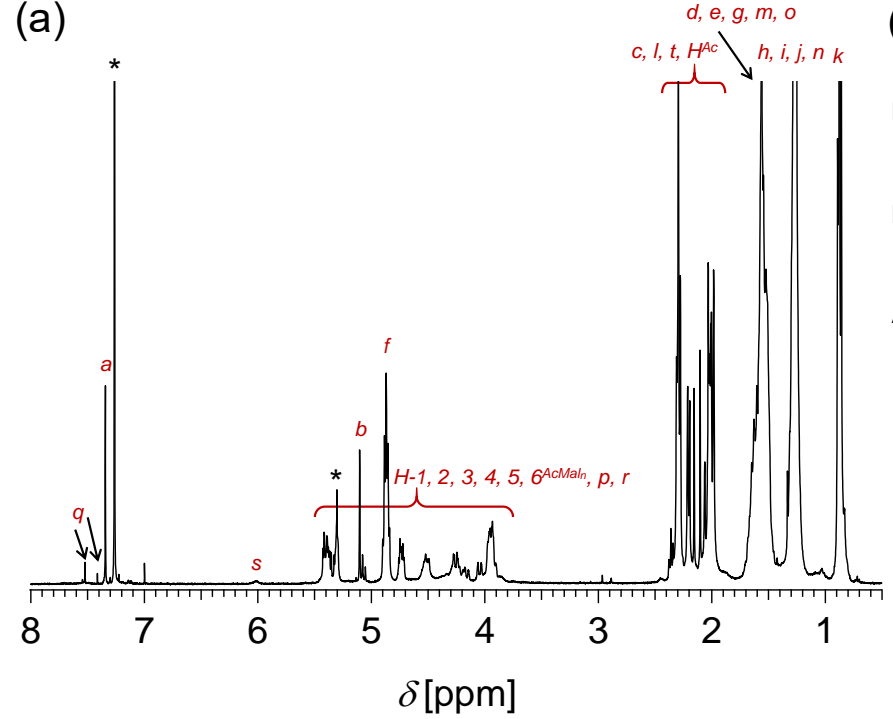

(b)

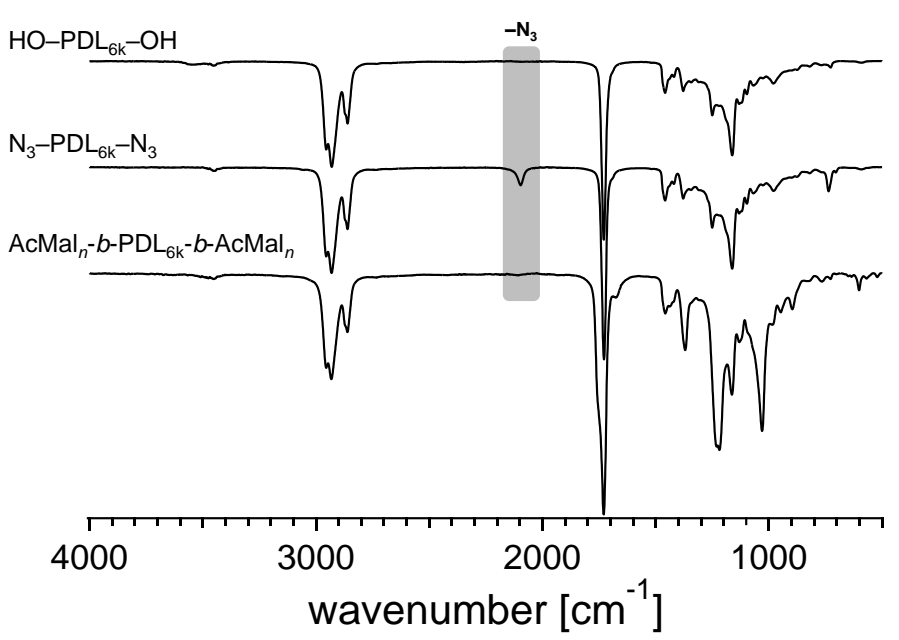

Figure S12. Characterization of $\mathrm{AcMal}_{n}-b-\mathrm{PDL}_{6 \mathrm{k}}-b-\mathrm{AcMal}_{n}$. (a) ${ }^{1} \mathrm{H}$ NMR spectrum $\left(\mathrm{CDCl}_{3}, 400 \mathrm{MHz}\right)$. (b) FT-IR spectra of $\mathrm{HO}-\mathrm{PDL}_{6 \mathrm{k}}-\mathrm{OH}$ (upper), $\mathrm{N}_{3}-\mathrm{PDL}_{6 \mathrm{k}}-\mathrm{N}_{3}$ (middle) and $\mathrm{AcMal}_{n}-b-\mathrm{PDL}_{6 \mathrm{k}}-b-\mathrm{AcMal}_{n}$ (lower). 


\section{Synthesis of $\mathrm{AcMal}_{n}-b-\mathrm{PDL}_{10 \mathrm{k}}-b-\mathrm{AcMal}_{n}$}

The Method C was used for the click reaction of $\mathrm{N}_{3}-\mathrm{PDL}_{10 \mathrm{k}}-\mathrm{N}_{3}\left(M_{\mathrm{n}, \mathrm{NMR}}=10,300 \mathrm{~g} \mathrm{~mol}^{-1}, 1.00 \mathrm{~g}\right.$, $97.0 \mu \mathrm{mol})$ and $\mathrm{AcMal}_{n}-\mathrm{C} \equiv \mathrm{CH}(492 \mathrm{mg}, 252 \mu \mathrm{mol})$ in DMF $(10 \mathrm{~mL})$ with $\mathrm{CuBr}(39 \mathrm{mg}, 0.27 \mathrm{mmol})$ and $\operatorname{PMDETA}(57 \mu \mathrm{L}, 0.27 \mathrm{mmol})$ to synthesize $\mathrm{AcMal}_{n}-b-\mathrm{PDL}_{10 \mathrm{k}}-b-\mathrm{AcMal}_{n}$. The crude product was purified by the preparative SEC to give $\mathrm{AcMal}_{n}-b-\mathrm{PDL}_{10 \mathrm{k}}-b-\mathrm{AcMal}_{n}$ as a yellow elastic material $(846 \mathrm{mg})$. Yield: $61.4 \%$. $M_{\mathrm{n}, \mathrm{SEC}}=17,000 \mathrm{~g} \mathrm{~mol}^{-1}(\mathrm{THF}), Ð=1.04(\mathrm{THF}), M_{\mathrm{n}, \mathrm{total}}=14,200 \mathrm{~g} \mathrm{~mol}^{-1}$.

${ }^{1} \mathrm{H}$ NMR (400 MHz, $\mathrm{CDCl}_{3}$ ): $\delta$ (ppm) 7.52, 7.41 (2x s, rotamers, triazole methine), 7.34 (s, aromatic), 6.045.99 (br, $-\mathrm{CH}(\mathrm{NAc})-), 5.48-3.77\left(\mathrm{~m},-\mathrm{Ph}-\mathrm{CH}_{2} \mathrm{O}-,-\mathrm{CH}_{2} \mathrm{CH}\left(\mathrm{C}_{5} \mathrm{H}_{11}\right) \mathrm{O}-,-\mathrm{CH}_{2} \mathrm{CH}_{2}-\right.$ triazole- $, \mathrm{H}-1,2,-3,-4$, $\left.-5,-6^{\mathrm{AcMal} n},-\mathrm{N}(\mathrm{Ac}) \mathrm{CH}_{2}-\right), 2.41-1.80\left(\mathrm{~m},-(\mathrm{C}=\mathrm{O}) \mathrm{CH}_{2} \mathrm{CH}_{2}-, \mathrm{CH}_{3}\right.$ in $\left.\mathrm{Ac}^{\mathrm{AcMal} n},-\mathrm{N}(\mathrm{C}=\mathrm{O}) \mathrm{CH}_{3}\right), 1.75-1.41(\mathrm{~m}$, main chain $-\left(\mathrm{CH}_{2}\right)_{2} \mathrm{CH}\left(\mathrm{CH}_{2}\left(\mathrm{CH}_{2}\right)_{3} \mathrm{CH}_{3}\right) \mathrm{O}-, \quad-\mathrm{CH}_{2} \mathrm{CH}_{2} \mathrm{CH}_{2} \mathrm{CH}_{2}-$ triazole), $1.35-0.98 \quad$ (m, $-\mathrm{CH}\left(\mathrm{CH}_{2}\left(\mathrm{CH}_{2}\right)_{3} \mathrm{CH}_{3}\right) \mathrm{O}-,-\mathrm{CH}_{2}\left(\mathrm{CH}_{2}\right)_{2}-$ triazole $), 0.88\left(\mathrm{t}, J=6.8 \mathrm{~Hz},-\mathrm{CH}_{3}\right)$. 

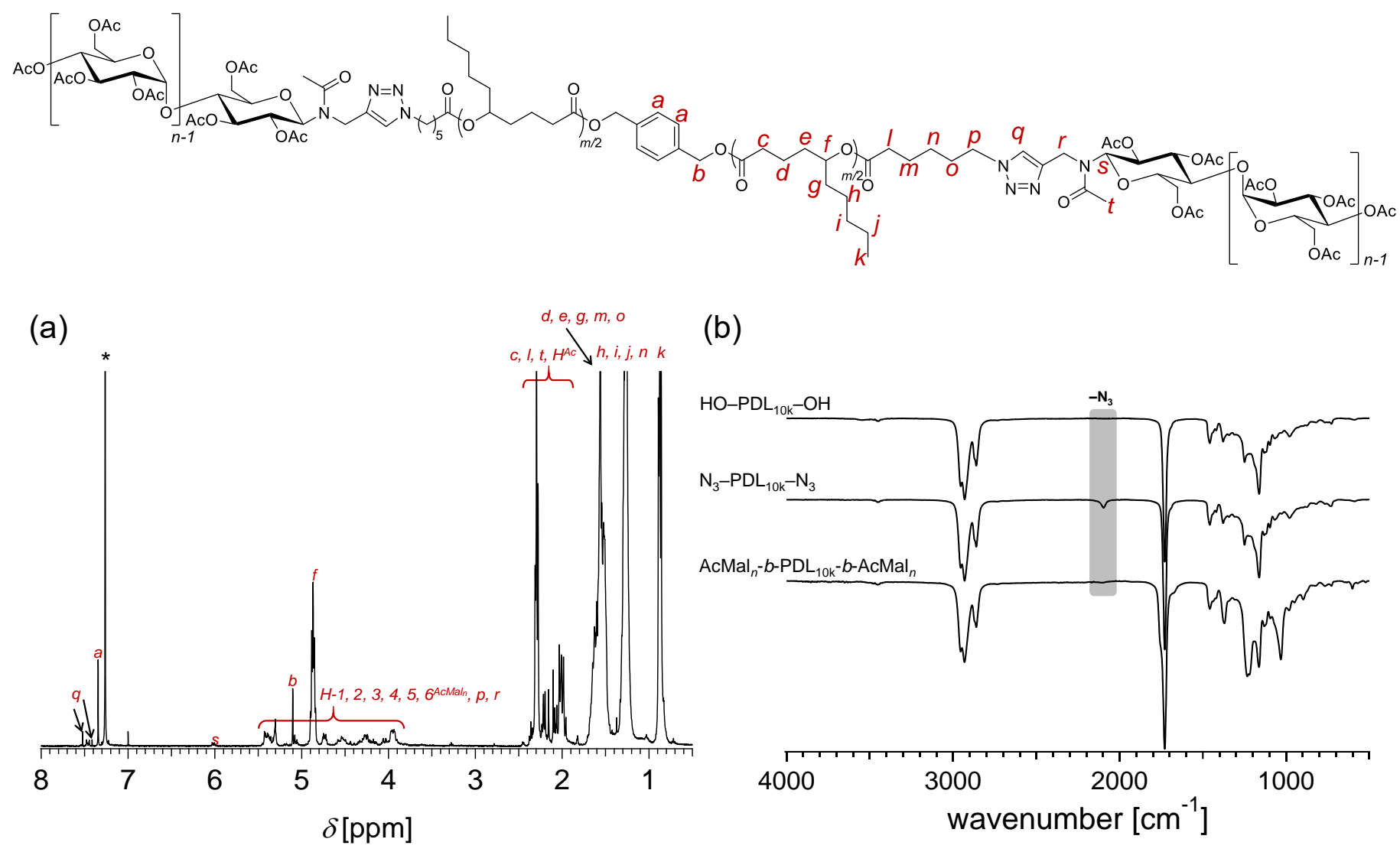

(b)

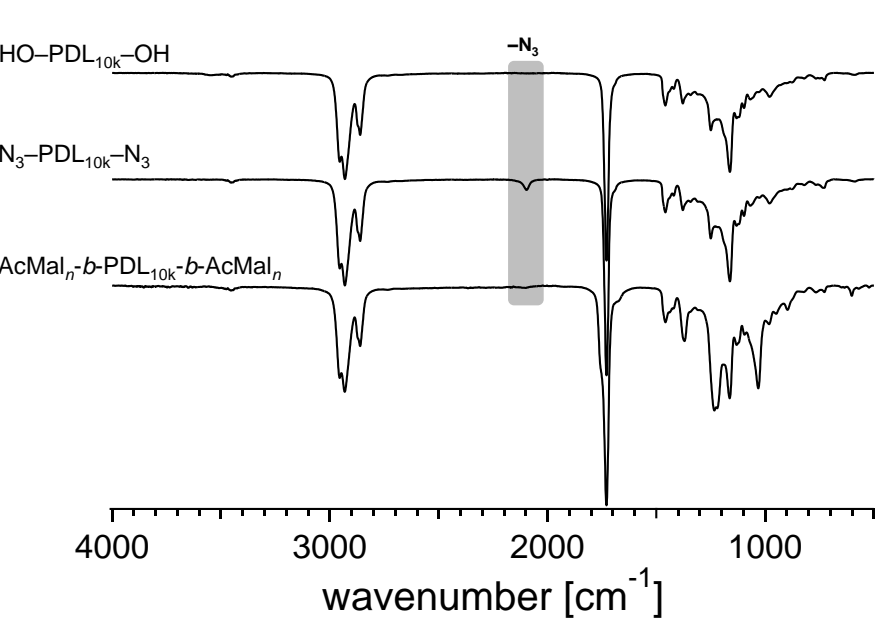

Figure S13. Characterization of $\mathrm{AcMal}_{n}-b-\mathrm{PDL}_{10 \mathrm{k}}-b-\mathrm{AcMal}_{n}$. (a) ${ }^{1} \mathrm{H} \mathrm{NMR}$ spectrum $\left(\mathrm{CDCl}_{3}, 400 \mathrm{MHz}\right)$. (b) FT-IR spectra of $\mathrm{HO}-\mathrm{PDL}_{10 \mathrm{k}}-\mathrm{OH}$ (upper), $\mathrm{N}_{3}-\mathrm{PDL}_{10 \mathrm{k}}-\mathrm{N}_{3}$ (middle), and $\mathrm{AcMal}_{n}-b-\mathrm{PDL}_{10 \mathrm{k}}-b-\mathrm{AcMal}_{n}($ lower). 


\section{Synthesis of $\mathrm{AcMal}_{n}-b-\mathrm{PDL}_{22 \mathrm{k}}-b-\mathrm{AcMal}_{n}$}

The Method C was used for the click reaction of $\mathrm{N}_{3}-\mathrm{PDL}_{22 \mathrm{k}}-\mathrm{N}_{3}\left(M_{\mathrm{n}, \mathrm{NMR}}=21,900 \mathrm{~g} \mathrm{~mol}^{-1}, 1.00 \mathrm{~g}\right.$, $46.0 \mu \mathrm{mol})$ and $\mathrm{AcMal}_{n}-\mathrm{C} \equiv \mathrm{CH}(235 \mathrm{mg}, 120 \mu \mathrm{mol})$ in $\mathrm{DMF}(10 \mathrm{~mL})$ with $\mathrm{CuBr}(23 \mathrm{mg}, 0.16 \mathrm{mmol})$ and PMDETA $(34 \mu \mathrm{L}, 0.16 \mathrm{mmol})$. The crude product was purified by the preparative SEC to give $\mathrm{AcMal}_{n}-b$ $\mathrm{PDL}_{22 \mathrm{k}}-b-\mathrm{AcMal}_{n}$ as a yellow elastic material (309 mg). Yield: $26.2 \%$.

$M_{\mathrm{n}, \mathrm{SEC}}=28,300 \mathrm{~g} \mathrm{~mol}^{-1}(\mathrm{THF}), Ð=1.04(\mathrm{THF}), M_{\mathrm{n}, \mathrm{total}}=25,800 \mathrm{~g} \mathrm{~mol}^{-1}$.

${ }^{1} \mathrm{H}$ NMR (400 MHz, $\mathrm{CDCl}_{3}$ ): $\delta$ (ppm) 7.52, 7.41 (2x s, rotamers, triazole methine), 7.34 (s, aromatic), 6.045.99 (br, $-\mathrm{CH}(\mathrm{NAc})-), 5.45-3.55\left(\mathrm{~m},-\mathrm{Ph}-\mathrm{CH}_{2} \mathrm{O}-,-\mathrm{CH}_{2} \mathrm{CH}\left(\mathrm{C}_{5} \mathrm{H}_{11}\right) \mathrm{O}-,-\mathrm{CH}_{2} \mathrm{CH}_{2}-\right.$ triazole- $, \mathrm{H}-1,2,-3,-4$, $\left.-5,-6^{\mathrm{AcMal} n},-\mathrm{N}(\mathrm{Ac}) \mathrm{CH}_{2}-\right), 2.49-1.84\left(\mathrm{~m},-(\mathrm{C}=\mathrm{O}) \mathrm{CH}_{2} \mathrm{CH}_{2}-, \mathrm{CH}_{3}\right.$ in $\left.\mathrm{Ac} \mathrm{AcMal} n^{\mathrm{Ac}}, \mathrm{N}(\mathrm{C}=\mathrm{O}) \mathrm{CH}_{3}\right), 1.84-1.40,(\mathrm{~m}$, main chain $-\left(\mathrm{CH}_{2}\right)_{2} \mathrm{CH}\left(\mathrm{CH}_{2}\left(\mathrm{CH}_{2}\right)_{3} \mathrm{CH}_{3}\right) \mathrm{O}-, \quad-\mathrm{CH}_{2} \mathrm{CH}_{2} \mathrm{CH}_{2} \mathrm{CH}_{2}-$ triazole), $\quad 1.34-0.99 \quad$ (m, $-\mathrm{CH}\left(\mathrm{CH}_{2}\left(\mathrm{CH}_{2}\right)_{3} \mathrm{CH}_{3}\right) \mathrm{O}-,-\mathrm{CH}_{2}\left(\mathrm{CH}_{2}\right)_{2}$-triazole $), 0.88\left(\mathrm{t}, J=6.6 \mathrm{~Hz},-\mathrm{CH}_{3}\right)$. 

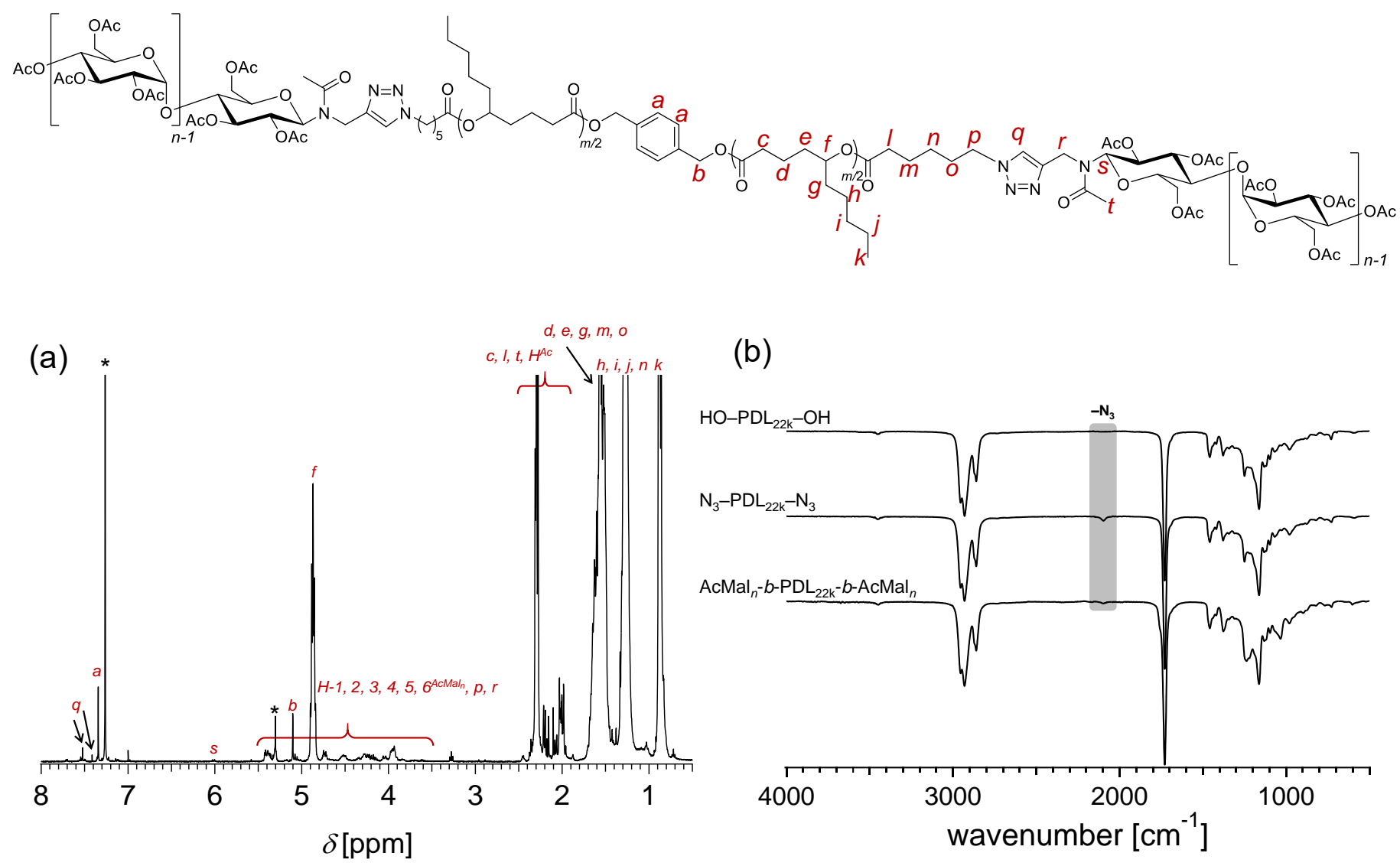

(b)

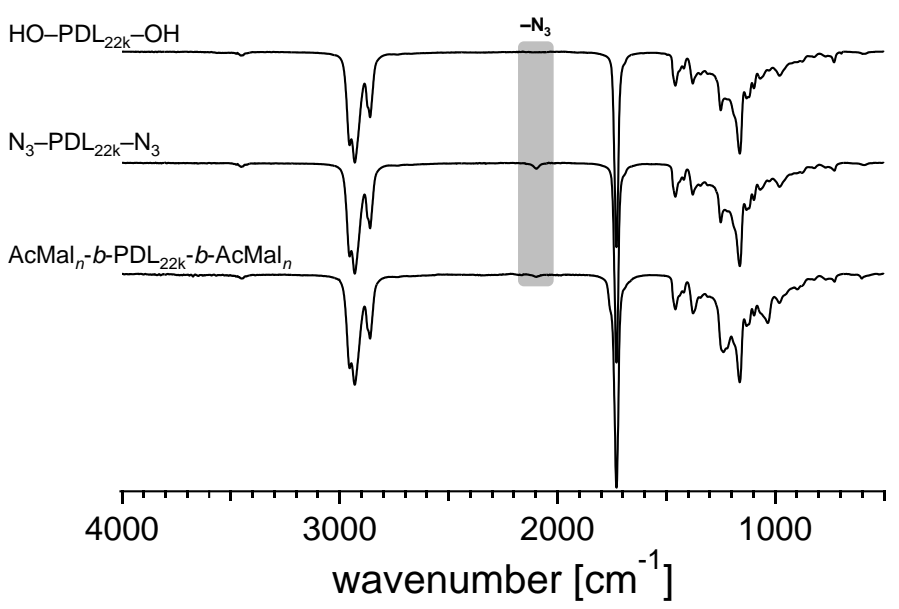

Figure S14. Characterization of $\mathrm{AcMal}_{n}-b-\mathrm{PDL}_{22 \mathrm{k}}-b-\mathrm{AcMal}_{n}$. (a) ${ }^{1} \mathrm{H}$ NMR spectrum $\left(\mathrm{CDCl}_{3}, 400 \mathrm{MHz}\right)$. (b) FT-IR spectra of $\mathrm{HO}-\mathrm{PDL}_{22 \mathrm{k}}-\mathrm{OH}$ (upper), $\mathrm{N}_{3}-\mathrm{PDL}_{22 \mathrm{k}}-\mathrm{N}_{3}$ (middle) and $\mathrm{AcMal}_{n}-b$ - $\mathrm{PDL}_{22 \mathrm{k}}-b$-AcMal ${ }_{n}$ (lower). 
(a) AcCel- $b$-PDL ${ }_{6 k}-b-$ AcCel

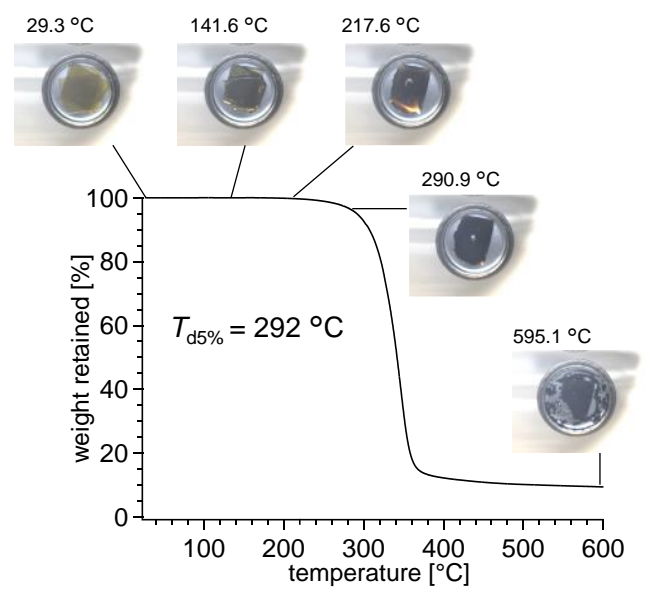

(d) AcMal-b-PDL $6 \mathrm{k}-b$-AcMal

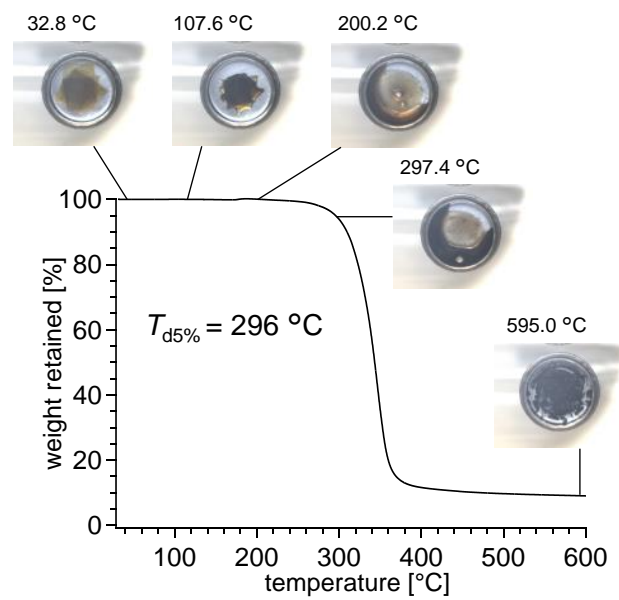

(b) AcCel-b-PDL ${ }_{10 k}-b-A c C e l$

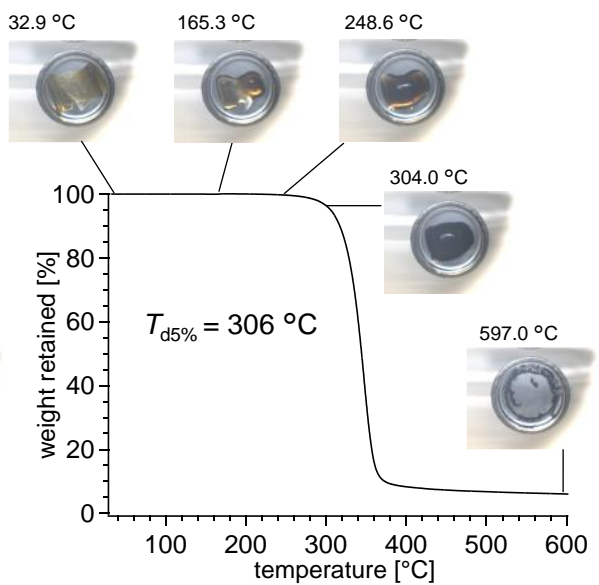

(e) AcMal- $b-\mathrm{PDL}_{10 \mathrm{k}}-b$-AcMal

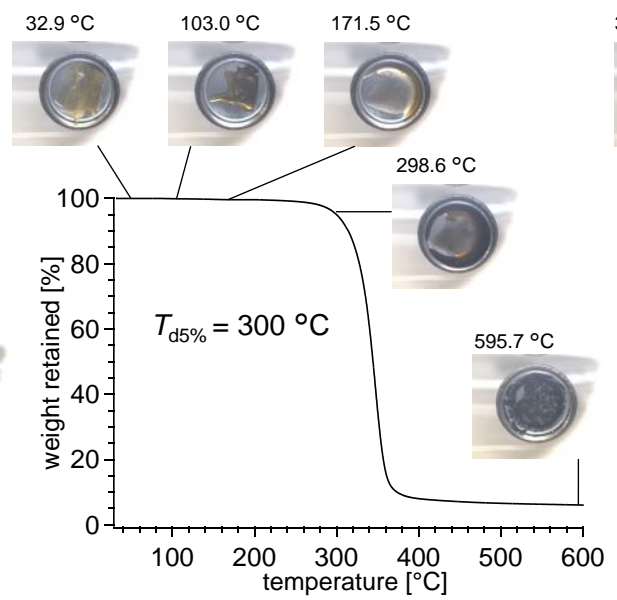

(c) AcCel- $b-\mathrm{PDL}_{22 \mathrm{k}}-b-\mathrm{AcCel}$

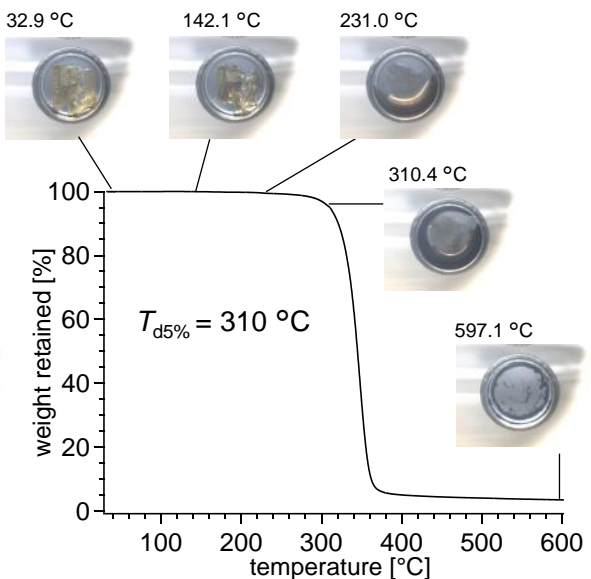

(f) AcMal- $b$-PDL ${ }_{10 k}-b-A c M a l$

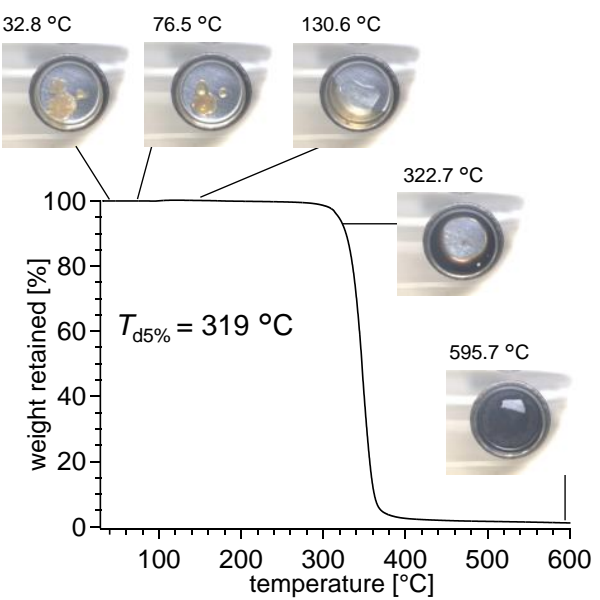

Figure S15. TGA curves of (a) $\mathrm{AcCel}_{n}-\mathrm{PDL}_{6 \mathrm{k}}-\mathrm{AcCel}_{n}$, (b) $\mathrm{AcCel}_{n}-\mathrm{PDL}_{10 \mathrm{k}}-\mathrm{AcCel}_{n}$, (c) $\mathrm{AcCel}_{n}-\mathrm{PDL}_{22 \mathrm{k}}-\mathrm{AcCel}_{n}$, (d) $\mathrm{AcMal}_{n}-\mathrm{PDL}_{6 \mathrm{k}}-\mathrm{AcMal}_{n}$, (e) $\mathrm{AcMal}_{n}-\mathrm{PDL}_{10 \mathrm{k}}-\mathrm{AcMal}_{n}$, and (f) $\mathrm{AcMal}_{n}-\mathrm{PDL}_{22 \mathrm{k}}-\mathrm{AcMal}_{n}$ (under nitrogen atmosphere; heating rate, $\left.10{ }^{\circ} \mathrm{C} \mathrm{min}^{-1}\right)$. Inset photographs show the sample appearance at the indicated temperature. $T_{\mathrm{d} 5 \%}$ means $5 \%$ weight loss temperature. 
(a) $\mathrm{N}_{3}-\mathrm{PDL}_{6 \mathrm{k}}-\mathrm{N}_{3}$

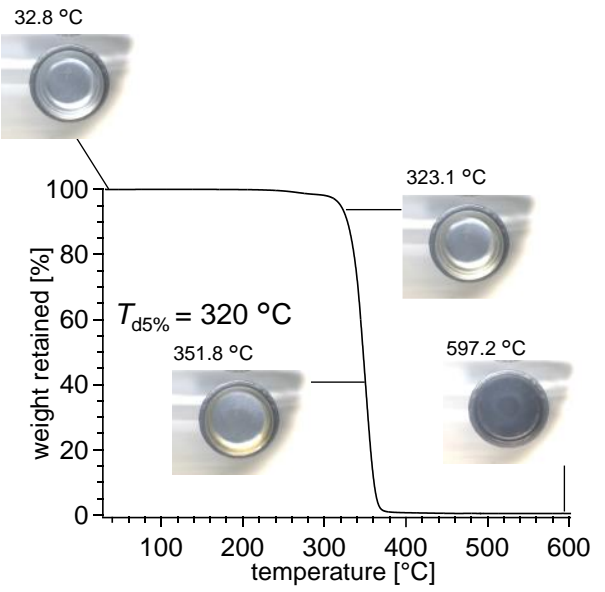

(d) $\mathrm{AcCel}_{n}-\mathrm{C} \equiv \mathrm{CH}$

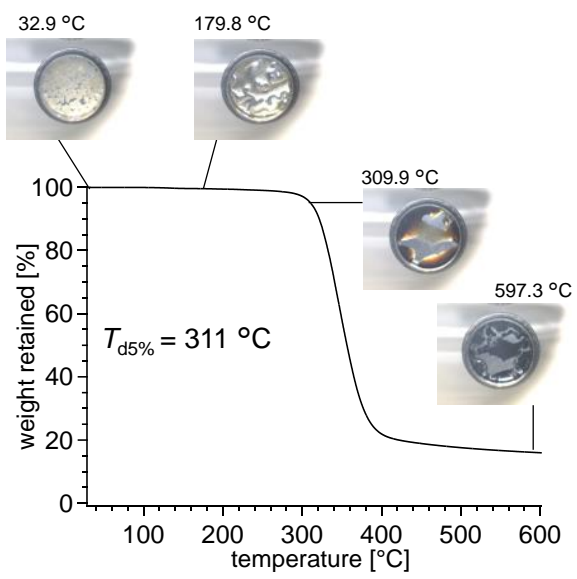

(b) $\mathrm{N}_{3}-\mathrm{PDL}_{10 \mathrm{k}}-\mathrm{N}_{3}$

$32.8^{\circ} \mathrm{C}$

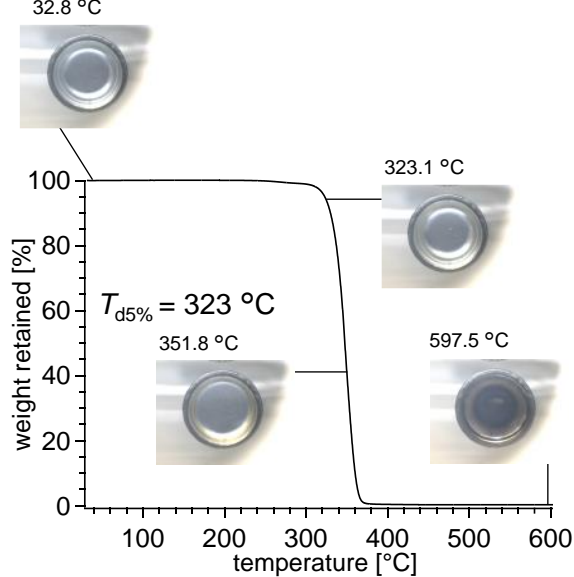

(e) $\mathrm{AcMal}_{n}-\mathrm{C} \equiv \mathrm{CH}$

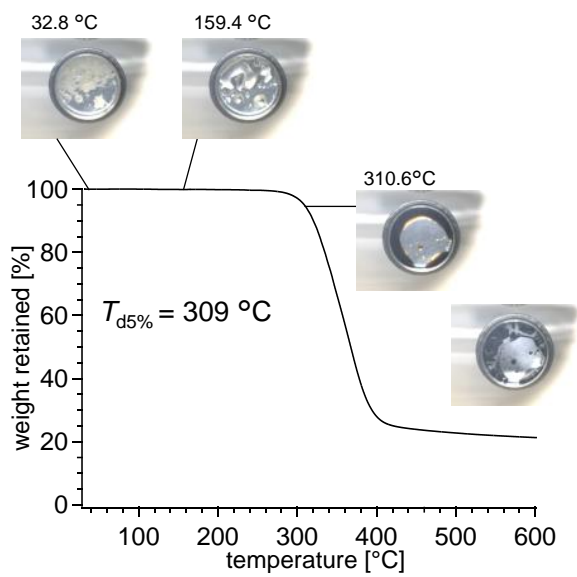

(c) $\mathrm{N}_{3}-\mathrm{PDL}_{22 \mathrm{k}}-\mathrm{N}_{3}$

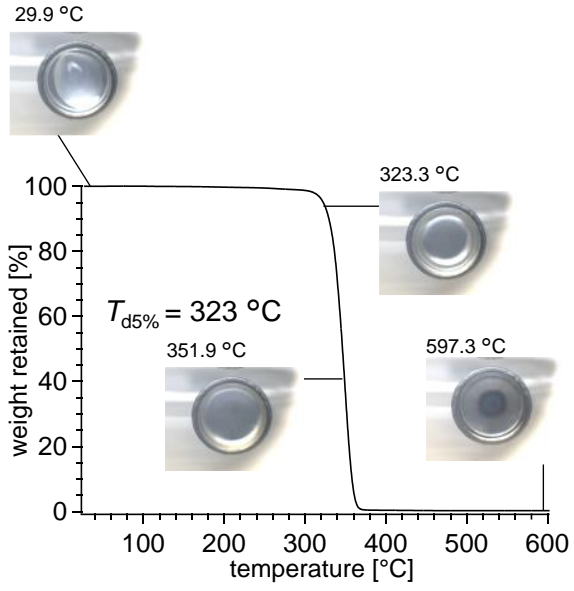

Figure S16. TGA curves of (a) $\mathrm{N}_{3}-\mathrm{PDL}_{6 \mathrm{k}}-\mathrm{N}_{3}$, (b) $\mathrm{N}_{3}-\mathrm{PDL}_{10 \mathrm{k}}-\mathrm{N}_{3}$, (c) $\mathrm{N}_{3}-\mathrm{PDL}_{22 \mathrm{k}}-\mathrm{N}_{3}$, (d) $\mathrm{AcCel}_{n}-\mathrm{C} \equiv \mathrm{CH}$, and (e) $\mathrm{AcMal}_{n}-\mathrm{C} \equiv \mathrm{CH}$ (under nitrogen atmosphere; heating rate, $10{ }^{\circ} \mathrm{C} \mathrm{min}^{-1}$ ). Inset photographs show the sample appearance at the indicated temperature. $T_{\mathrm{d} 5 \%}$ means $5 \%$ weight loss temperature. 


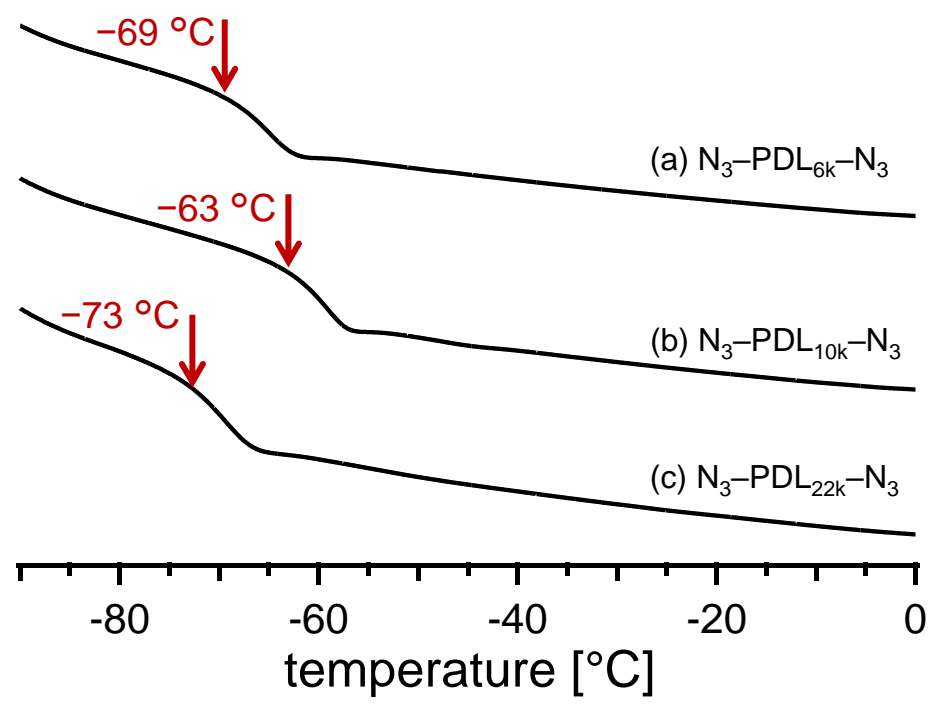

Figure S17. DSC curves traces of (a) $\mathrm{N}_{3}-\mathrm{PDL}_{6 \mathrm{k}}-\mathrm{N}_{3}$, (b) $\mathrm{N}_{3}-\mathrm{PDL}_{10 \mathrm{k}}-\mathrm{N}_{3}$, and (c) $\mathrm{N}_{3}-\mathrm{PDL}_{22 \mathrm{k}}-\mathrm{N}_{3}$ during 2 nd heating run.

(a) $\mathrm{AcCel}_{n}-\mathrm{C} \equiv \mathrm{CH}$

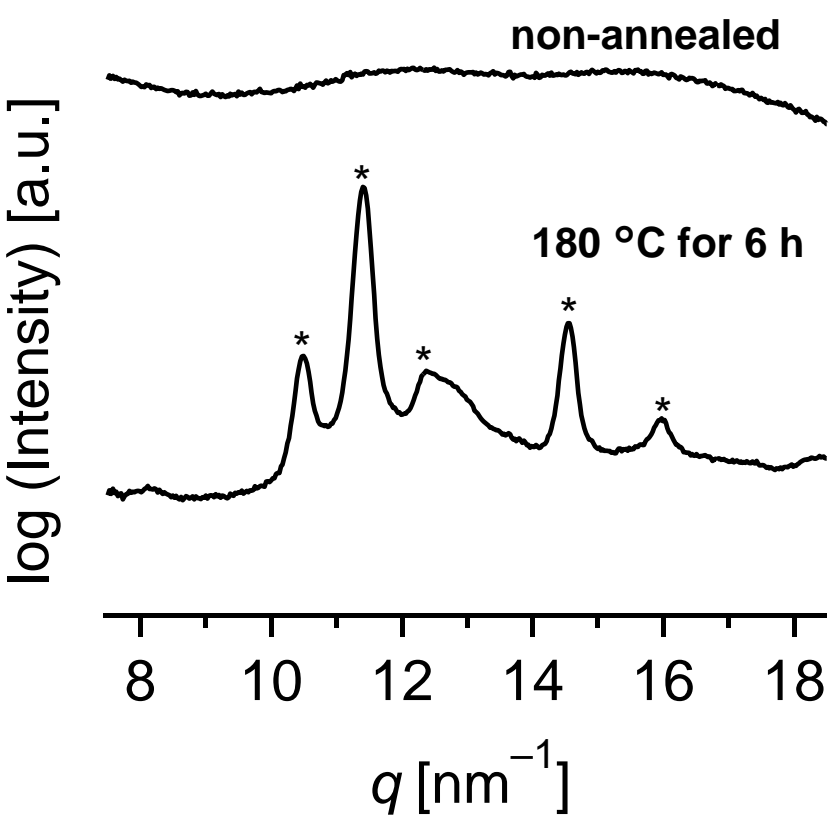

(b) $\mathrm{AcMal}_{n}-\mathrm{C} \equiv \mathrm{CH}$

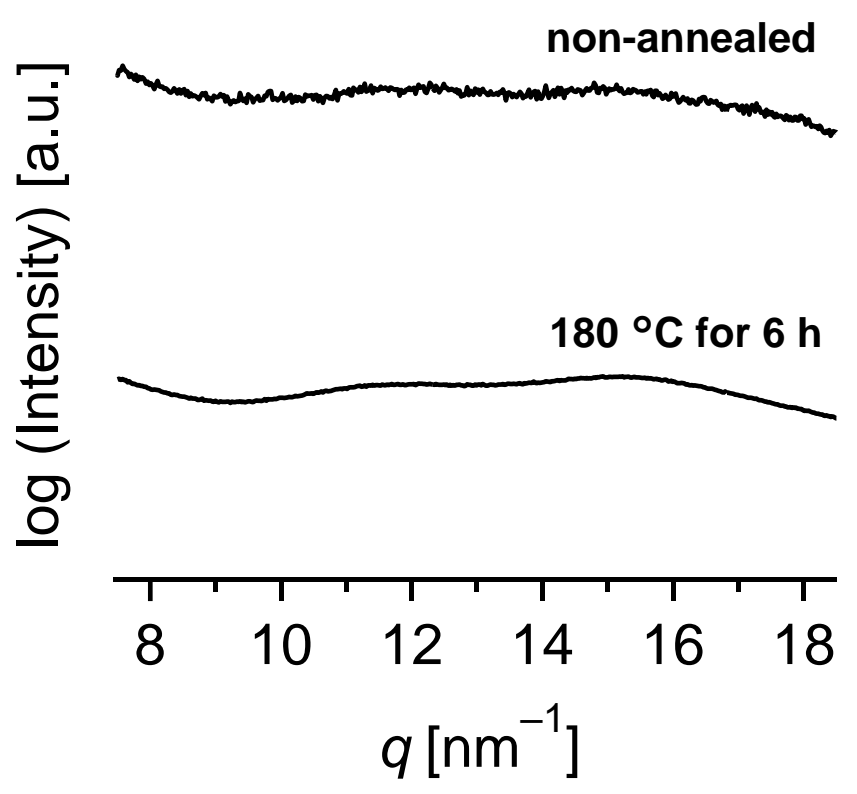

Figure S18. WAXD profiles of (a) $\mathrm{AcCel}_{n}-\mathrm{C} \equiv \mathrm{CH}$ and (b) $\mathrm{AcMal}_{n}-\mathrm{C} \equiv \mathrm{CH}$ without thermal annealing and after thermal annealing at $180^{\circ} \mathrm{C}$ for $6 \mathrm{~h}$. The asterisks denote the diffraction peak from cellulose triacetate I crystal. 
(a) $\mathrm{AcCel}_{n}-b-\mathrm{PDL}_{6 \mathrm{k}}-b-\mathrm{AcCel}_{n}$

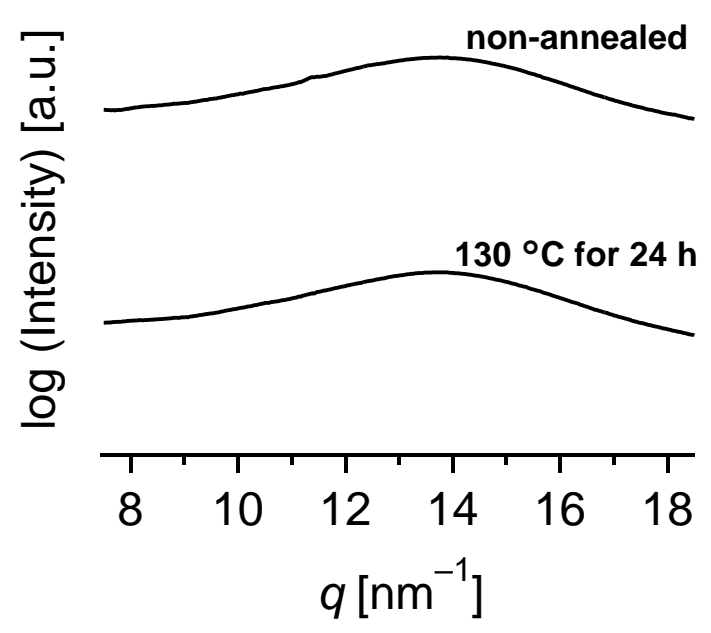

(c) $\mathrm{AcCel}_{n}-b-\mathrm{PDL}_{10 \mathrm{k}}-b-\mathrm{AcCel}_{n}$

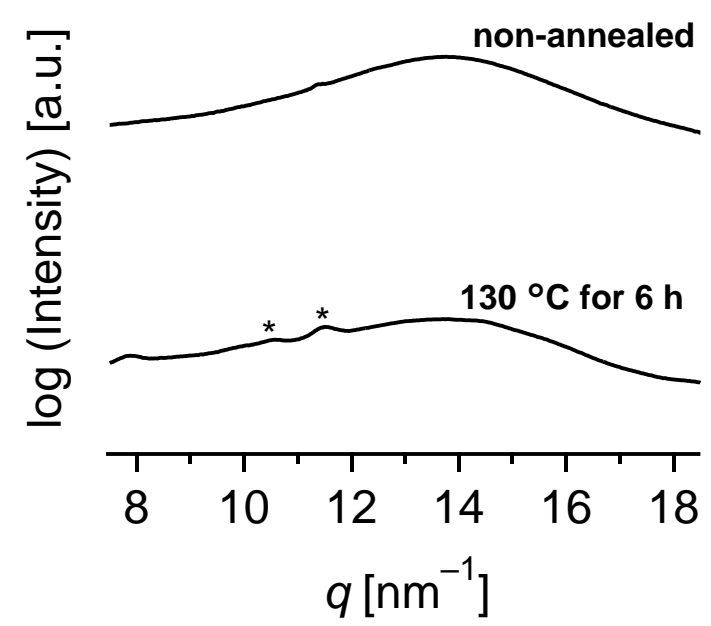

(e) $\mathrm{AcCel}_{n}-b-\mathrm{PDL}_{22 \mathrm{k}}-b-\mathrm{AcCel}_{n}$

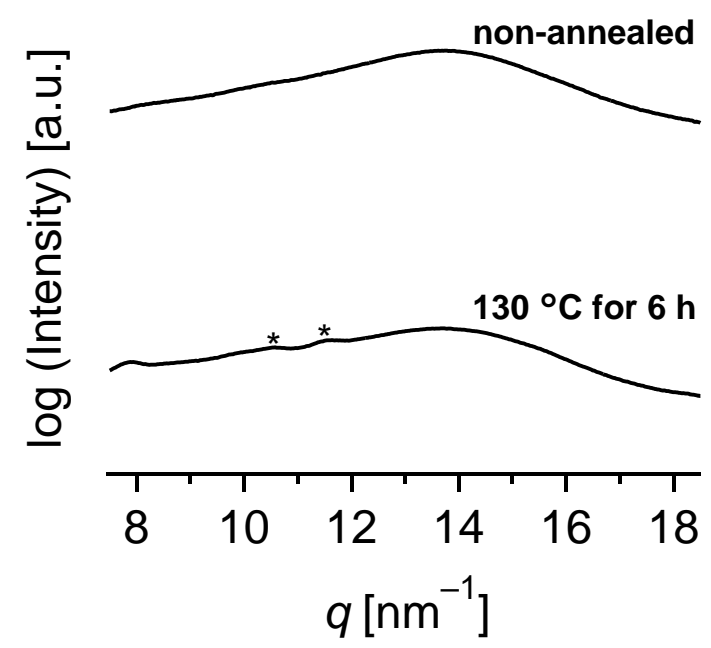

(b) $\mathrm{AcMal}_{n}-b-\mathrm{PDL}_{6 \mathrm{k}}-b-\mathrm{AcMal}_{n}$

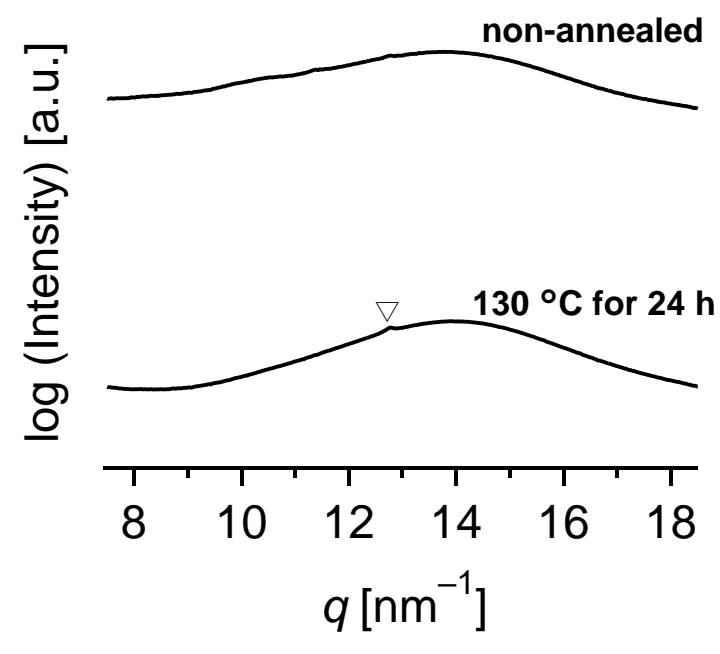

(d) $\mathrm{AcMal}_{n}-b-\mathrm{PDL}_{10 \mathrm{k}}-\mathrm{b}-\mathrm{AcMal}_{n}$

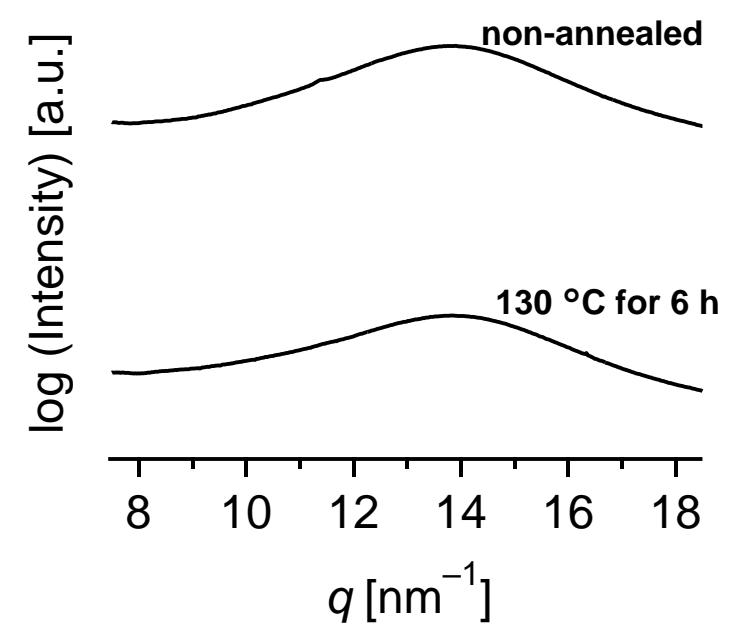

(f) $\mathrm{AcMal}_{n}-b-\mathrm{PDL}_{22 \mathrm{k}}-b-\mathrm{AcMal}_{n}$

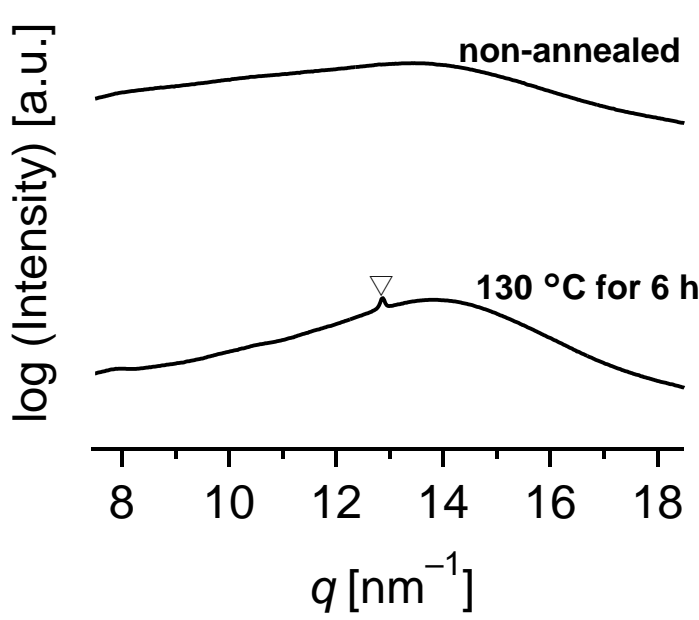

Figure S19. WAXD profiles of (a) $\mathrm{AcCel}_{n}-b-\mathrm{PDL}_{6 \mathrm{k}}-b-\mathrm{AcCel}_{n}$, (b) $\mathrm{AcMal}_{n}-b-\mathrm{PDL}_{6 \mathrm{k}}-b-\mathrm{AcMal}_{n}$, (c) $\mathrm{AcCel}_{n}-b-$ $\mathrm{PDL}_{10 \mathrm{k}}-b-\mathrm{AcCel}_{n}$, (d) $\mathrm{AcMal}_{n}-b-\mathrm{PDL}_{10 \mathrm{k}}-b-\mathrm{AcMal}_{n}$, (e) $\mathrm{AcCel}_{n}-b-\mathrm{PDL}_{22 \mathrm{k}}-b-\mathrm{AcCel}_{n}$, and (f) $\mathrm{AcMal}_{n}-b-\mathrm{PDL}_{22 \mathrm{k}}-$ $b$-AcMal ${ }_{n}$ without thermal annealing and after thermal annealing at $130{ }^{\circ} \mathrm{C}$ for 6 or $24 \mathrm{~h}$. The asterisks denote the diffraction peak from cellulose triacetate I crystal and inverted triangles denote artifact peak. 
(a) AcCel-b-PDL ${ }_{6 k}-b-A c C e l$

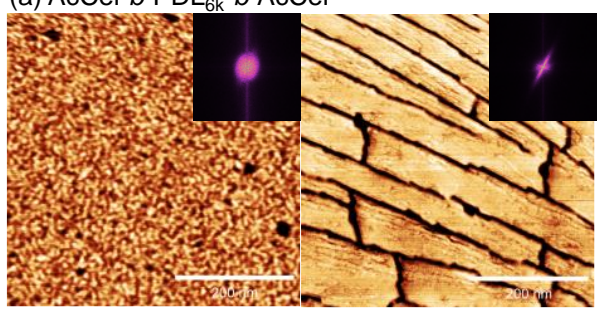

(d) AcMal- $b-\mathrm{PDL}_{6 \mathrm{k}}-b-\mathrm{AcMal}$

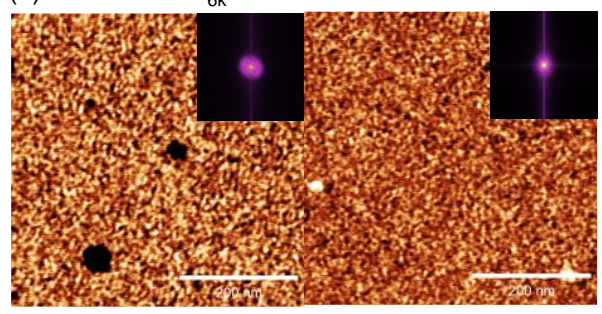

(b) AcCel- $b-\mathrm{PDL}_{10 \mathrm{k}}-b-\mathrm{AcCel}$

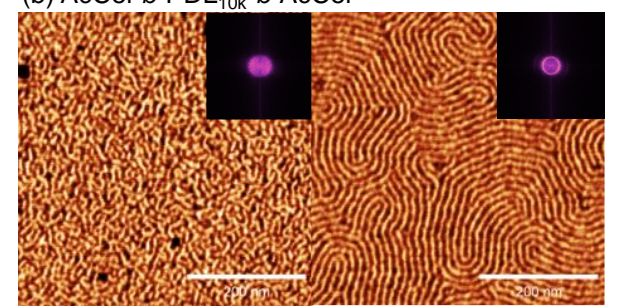

(e) AcMal-b-PDL $10 k-b-$ AcMal

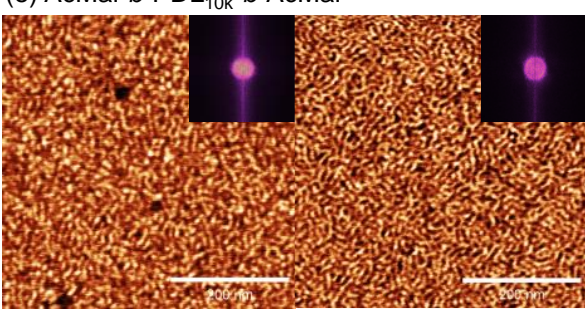

(c) AcCel- $b-\mathrm{PDL}_{22 k}-b-\mathrm{AcCel}$

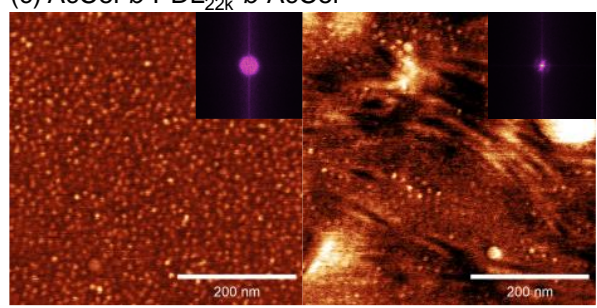

(f) AcMal- $b-\mathrm{PDL}_{22 \mathrm{k}}-b-\mathrm{AcMal}$

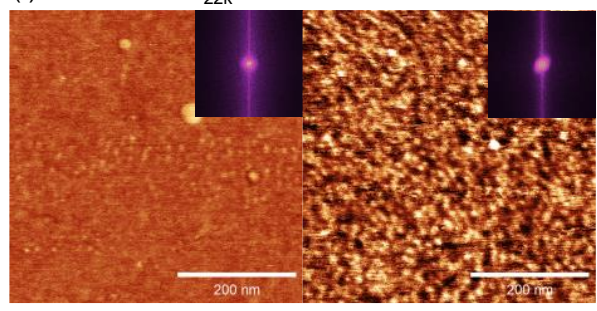

Figure S20. AFM phase images of (a) $\mathrm{AcCel}_{n}-b-\mathrm{PDL}_{6 \mathrm{k}}-b-\mathrm{AcCel}_{n}$, (b) $\mathrm{AcCel}_{n}-b-\mathrm{PDL}_{10 \mathrm{k}}-b-\mathrm{AcCel}_{n}$, (c) $\mathrm{AcCel}_{n^{-}}$ $b$-PDL $22 \mathrm{k}-b-\mathrm{AcCel}_{n}$, (d) $\mathrm{AcMal}_{n}-b-\mathrm{PDL}_{6 \mathrm{k}}-b-\mathrm{AcMal}_{n}$, (e) $\mathrm{AcMal}_{n}-b-\mathrm{PDL}_{10 \mathrm{k}}-b-\mathrm{AcMal}_{n}$, and (f) $\mathrm{AcMal}_{n}-b-$ $\mathrm{PDL}_{22 \mathrm{k}}-b-\mathrm{AcMal}_{n}$ thin film before (left) and after (right) thermal annealing at $130{ }^{\circ} \mathrm{C}$. The insets show the corresponding FFT profiles. Scale bars are $200 \mathrm{~nm}$.

(a) AcCel-b-PDL 6 - $b$-AcCel

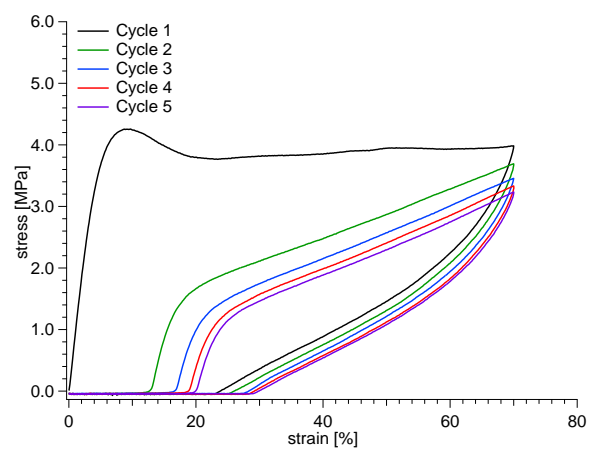

(d) AcMal- $b-\mathrm{PDL}_{6 \mathrm{k}}-b-\mathrm{AcMal}$

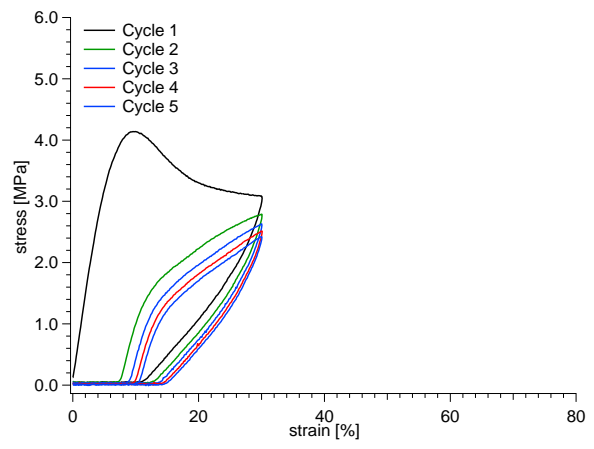

(b) AcCel-b-PDL $10 k-b-A c C e l$

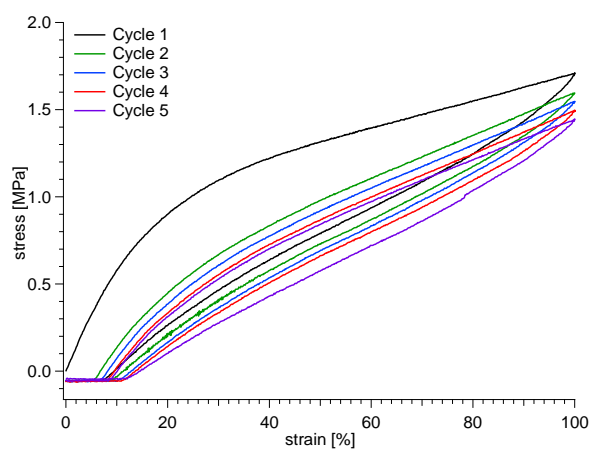

(e) AcMal-b-PDL ${ }_{10 \mathrm{k}}-b$-AcMal

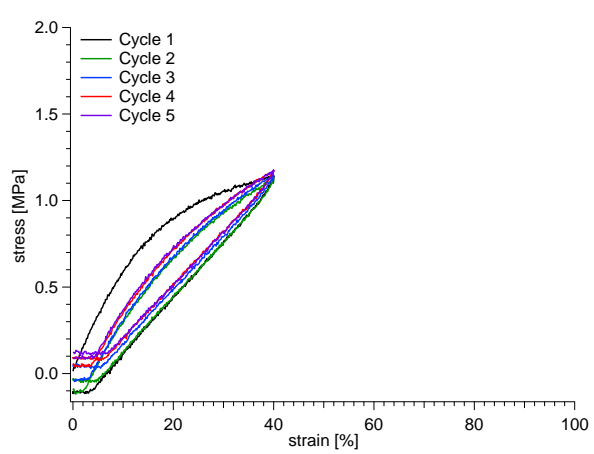

(c) AcCel- $b-\mathrm{PDL}_{22 \mathrm{k}}-b-\mathrm{AcCel}$

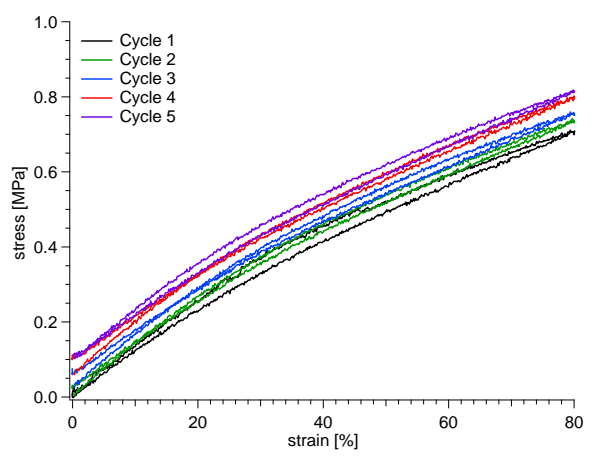

Figure S21. Cycle tensile testing (crosshead speed, $10 \mathrm{~mm} \mathrm{~min}^{-1}$ ) for (a) $\mathrm{AcCel}_{n}-b-\mathrm{PDL}_{6 \mathrm{k}}-b-\mathrm{AcCel}_{n}$, (b) $\mathrm{AcCel}_{n}-b-\mathrm{PDL}_{10 \mathrm{k}}-b-\mathrm{AcCel}_{n}$, (c) $\mathrm{AcCel}_{n}-b-\mathrm{PDL}_{22 \mathrm{k}}-b-\mathrm{AcCel}_{n}$, (d) $\mathrm{AcMal}_{n}-b-\mathrm{PDL}_{6 \mathrm{k}}-b-\mathrm{AcMal}_{n}$, and (e) $\mathrm{AcMal}_{n}-$ $b$-PDL $10 \mathrm{k}-b-\mathrm{AcMal}_{n}$. 
(a)

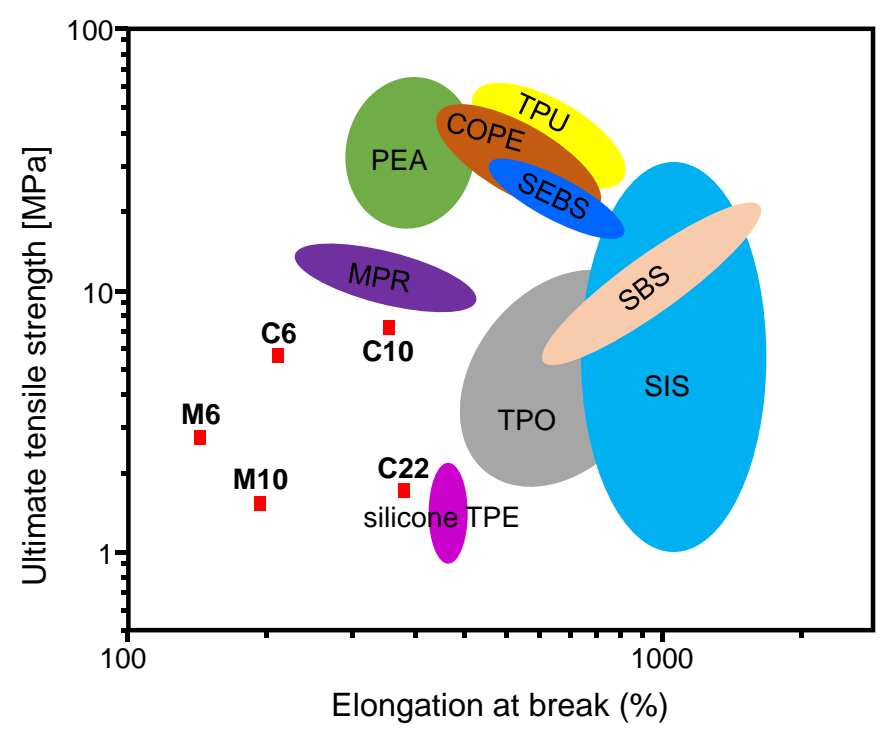

(b)

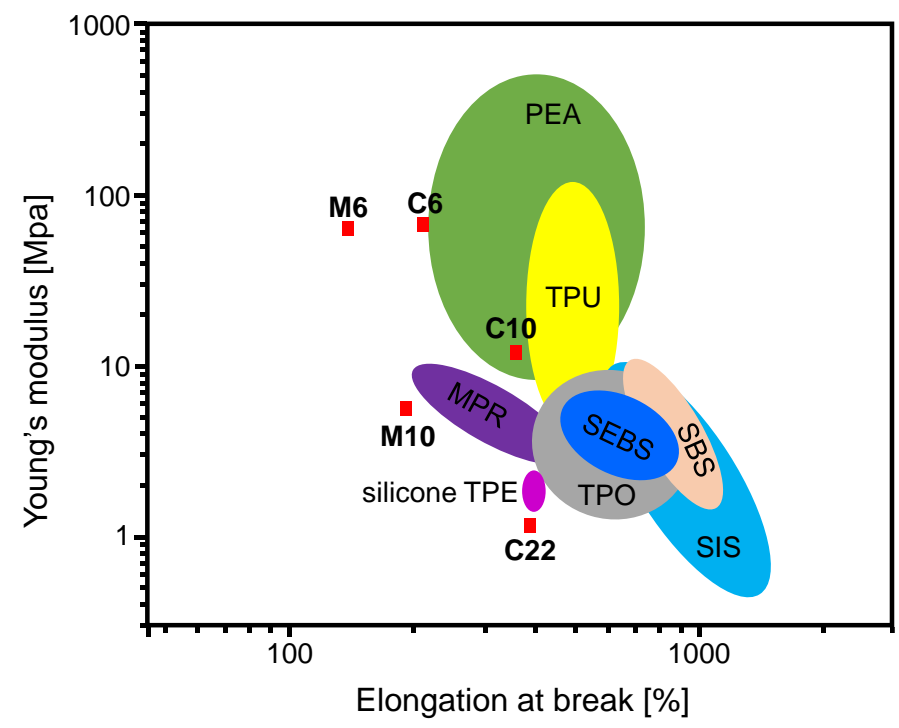

Figure S22. Comparison of (a) $\sigma_{\mathrm{b}}$ and (b) $E$ value of $\mathrm{AcCel}_{n}-b-\mathrm{PDL}_{6 \mathrm{k}}-b-\mathrm{AcCel}_{n}$ (C6), $\mathrm{AcCel}_{n}-b-\mathrm{PDL}_{10 \mathrm{k}}-b-$ $\mathrm{AcCel}_{n}(\mathrm{C} 10), \mathrm{AcCel}_{n}-b-\mathrm{PDL}_{22 \mathrm{k}}-b-\mathrm{AcCl}_{n}(\mathrm{C} 22), \mathrm{AcMal}_{n}-b-\mathrm{PDL}_{6 \mathrm{k}}-b-\mathrm{AcMal}_{n}(\mathrm{M} 6)$, and AcMal $n-b-\mathrm{PDL}_{10 \mathrm{k}}-b-$ $\mathrm{AcMal}_{n}$ (M10) with commercial TPEs. SBS, poly(styrene)- $b$-poly(butadiene)- $b$-poly(styrene); SIS, poly(styrene)- $b$-poly(isoprene)- $b$-poly(styrene); SEBS, poly(styrene)- $b$-poly(ethylene-co-butylene)- $b$ poly(styrene); PEA, TPEs with polyamide hard blocks; TPO, thermoplastic olefins; COPE, copolyester TPEs; MPR, melt processable rubber; TPU, thermoplastic polyurethanes. ${ }^{4}$

(1) Hiraishi, M.; Igarashi, K.; Kimura, S.; Wada, M.; Kitaoka, M.; Samejima, M. Synthesis of highly ordered cellulose II in vitro using cellodextrin phosphorylase. Carbohydr. Res. 2009, 344, 2468-2473.

(2) Grandjean, C.; Boutonnier, A.; Guerreiro, C.; Fournier, J. -M.; Mulard, L. A. On the Preparation of Carbohydrate-Protein Conjugates Using the Traceless Staudinger Ligation. J. Org. Chem. 2005, 70, 7123-7132.

(3) Halila, S.; Manguian, M.; Fort, S.; Cottaz, S.; Hamaide, T.; Fleury, E.; Driguez, H. Syntheses of WellDefined Glyco-Polyorganosiloxanes by "Click" Chemistry and Their Surfactant Properties. Macromol. Chem. Phys. 2008, 209, 1282-1290.

(4) Gregory, G. L.; Sulley, G. S.; Carrodeguas, L. P.; Chen, T. T. D.; Santmarti, A.; Terrill, N. J.; Lee, K. -Y.; Williams, C. K. Triblock polyester thermoplastic elastomers with semi-aromatic polymer end blocks by ring-opening copolymerization. Chem. Sci. 2020, 11, 6567-6581. 